\title{
ELM pacing and high-density operation using pellet injection in the ASDEX Upgrade all-metal-wall tokamak
}

\author{
P. T. Lang, A. Burckhart, M. Bernert, L. Casali, R. Fischer, O. Kardaun, G. Kocsis 1), \\ M. Maraschek, A. Mlynek, B. Plöckl, M. Reich, F. Ryter, J. Schweinzer, B. Sieglin, \\ W. Suttrop, T. Szepesi 1), G. Tardini, E. Wolfrum, D. Zasche, H. Zohm, \\ ASDEX Upgrade Team
}

MPI für Plasmaphysik, EURATOM Association., Boltzmannstr. 2, D-85748 Garching, Germany

1) Wigner RCP, RMI, EURATOM Association, P.O.Box 49, H-1525 Budapest-114, Hungary

\section{E-mail contact of main author: peter.lang@ipp.mpg.de}

\begin{abstract}
ELM triggering and pacing in an all-metal wall environment shows significant differences to a firstwall configuration containing carbon. Here we report on experiments performed at ASDEX Upgrade revisiting the issue with all plasma-facing surfaces now fully replaced by tungsten. This investigation was motivated by experimental findings indicating that ELM triggering becomes more intricate when the carbon is replaced by a metal wall. ELM pacing could no longer be achieved by magnetic triggering in ASDEX Upgrade under conditions that previously showed a positive response. Also, recent investigations at JET indicate that a lag time occurs in pellet ELM triggering when operating with the new ITER-like wall. The ASDEX Upgrade centrifugebased launching system was revitalized and upgraded for this study, now allowing detailed analysis of the ELM trigger response. The appearance of a lag time for pellet ELM triggering in an all-metal wall environment was confirmed. While different lag time durations were found for several type-I ELMy H-mode scenarios, the magnitude of the pellet perturbation was found to cause no difference. Reducing the auxiliary heating power for ELM triggering clearly makes the pellet tool less efficient for ELM control purposes; however, this affords a major benefit when applied for fuelling. Plasma operation with benign ELM behaviour at core densities far beyond the Greenwald limit was demonstrated, this being fully reversible and not affecting the energy confinement.
\end{abstract}

\section{Introduction:}

Injection of cryogenic solid hydrogen isotope pellets has turned out to be a powerful tool for development and enhancement of plasma scenarios. Two main tasks were assigned to this tool in the past: control and mitigation of edge-localized modes (ELMs) and efficient particle fuelling in order to achieve high-density operation while maintaining high confinement. Continued interest has been maintained for decades in "classical" pellet fuelling since it is more efficient than gas puffing. In particular, it was often found in present-day tokamaks when gas puff fuelling is used that density profiles become flat and are restricted to values below the Greenwald density $\mathrm{n}_{\mathrm{Gw}}$ which was derived as a disruptive density limit scaling in low confinement mode (L-mode) discharges [1]. However, spontaneous density profile peaking observed under specific conditions at DIII-D resulted in operation above the Greenwald density while maintaining good energy confinement [2,3]. Pellets penetrating sufficiently deep into the core plasma can smoothly overcome this limitation on demand. Studies for future fusion power plants based on the tokamak principle recommend operation at high densities above $\mathrm{n}_{\mathrm{Gw}}$ using peaked density profiles to avoid temperatures exceeding the optimum for D-T fusion at high central pressure and to facilitate the exhaust issue. While these peaked profiles are only accessible with pellet fuelling at the high collisionality of present-day tokamaks, it is predicted that they will occur naturally at the low collisonality in ITER and DEMO [4]. 
High fusion performance operation in ITER is based on the achievement of the high confinement mode (H-mode) regime with an edge transport barrier that is expected to lead to the quasi-periodic occurrence of ELMs [5]. Understanding and theoretical interpretation of ELMs has been the subject of intense research over the last decades, with pioneering work [6,7] explaining the occurrence of ELMs and pedestal dynamics by the peeling-ballooning theory. It assumes a coupling between two type of magnetohydrodynamic (MHD) instabilities, the pressure gradient driven ballooning modes and the current driven peeling modes. According models describe e.g. well the pedestal structures in individual devices [8,9], the non-linear ELM evolution [10] and the correlation between pedestal parameters to the overall confinement [11]. Owing to the severe threat to ITER emerging from the power load deposited by type-I ELMs onto the first wall and divertor, the emphasis of pellet investigation has recently shifted more towards its application for ELM control. ELM pacing aims to reduce the ELM size by triggering the instability through an external perturbation at a higher rate than the natural ELM frequency. Modelling of ELM triggering by pellet injection has been performed with the non-linear MHD code JOREK upgraded to include an appropriate description of the pellet ablation physics [12]. Simulations show pellet triggering of ELMs can be caused by the toroidally localized high edge pressure regions produced by the localized deposition of particles by the pellet and their reheating by the plasma [13]. Hence, pellets are considered as appropriate actuator in almost all operational scenarios requiring ELM pacing. ELM pacing will probably already be needed in the current ramp-up phase of the non-nuclear phase of ITER when entering the H-mode with a heating power slightly above the transition threshold value. As such phases are prone to long ELM-free phases followed by a large ELM causing high power loads and plasma stability problems, the onset of ELM activity must immediately be enforced once the L-H transition has occurred [14]. With ITER operated in the low-current regime, a minimum ELM frequency must be sustained to avoid impurity accumulation. When approaching higher currents, this frequency requirement is finally exceeded by the pacing frequency imposed by the need to keep the ELM size below the hazard limit [15].

Since developed at ASDEX Upgrade (AUG) [16], the technique of ELM control by pellet pacing has been confirmed on other tokamaks such as DIII-D [17] and JET [18]. Recently, in DIII-D plasmas designed to match the ITER baseline configuration, an increase of the spontaneous ELM frequency by a factor of up to 12 was demonstrated, while the energy and particle fluxes to the divertor were reduced accordingly [19]. Ostensibly, this might lead to the conclusion that pellet pacing can be regarded as a well-matured and reliable control tool applicable in future fusion devices such as ITER and beyond. However, it must be recalled that most of these positive results were obtained in devices in which at least part of the plasma-facing components (PFC) had carbon (C) surfaces. When abandoning $\mathrm{C}$ in favour of a more reactor relevant all-metal wall, plasma operation faces additional restrictions [20]. In addition, the plasma performance significantly decreases [21] in some scenarios and the ELM dynamics show distinct changes. This is attributed to a significant change of the pedestal conditions, which are greatly affected by the wall material. As a consequence, it was necessary to re-evaluate the ELM pellet pacing technique in the altered configuration with an all-metal wall.

A reconnaissance experiment at AUG tried to re-establish ELM control by magnetic triggering [22] after changeover to the all-metal wall was completed. Here, a first indication was found for ELM triggering becoming more intricate in relation to the $C$ period. Since these results have not yet been published, they will be briefly reported in this paper to complete the picture. Dedicated experiments on pellet ELM pacing have been conducted at JET with the newly installed ITER-like wall (ILW) and compared with previous C-wall results [18]. These 
investigations afforded further indication of reduced trigger potential; in particular, the appearance of a lag time for triggering was detected. Data clearly show that the probability of a pellet triggering an ELM is dependent on the time elapsing since the previous ELM with a zero probability during the lag time. Dependence on the pellet speed and mass was also observed, but it remained unclear if this was due to corresponding variations of the resulting pellet perturbation or to the limited technical performance of the high-frequency pellet injector (HFPI) [23] falling short of delivering all pellets in viable conditions. Pellets are regarded as viable for ELM triggering when providing sufficient mass and speed allowing penetration to the pressure profile pedestal top [24,25]. Primarily, HFPI was built with its performance designed to meet all viability requirements for ELM control and mitigation after the ELM impact had been found to potentially damage the JET divertor [26]. It was recently improved in order to further approach the initial requirements [27], and further amendments are in progress to allow detailed experiments on ELM-trigger physics. In order to further these investigations and verify the JET findings, the AUG pellet-launching system was revitalized and upgraded to resume operation for ELM pacing and fuelling studies. Special emphasis was put on all requirements for detailed triggering investigations; in particular, on injecting pellets with a high delivery reliability and showing only small mass and speed scatter. With its accelerator unit based on a centrifuge principle, the AUG system is particularly suited to these needs [28]. The all-metal-wall tokamak AUG is now equipped with an inboard pellet injection system covering a wide parameter range. This configuration is most suitable for performing investigations on the underlying physics of pellet ELM pacing and fuelling, and in order to allow reliable extrapolation towards ITER.

\section{Set-up}

AUG is a medium-sized divertor tokamak (major radius $\mathrm{R}_{0}=1.65 \mathrm{~m}$, minor radius $\mathrm{a}_{0}=0.5 \mathrm{~m}$, plasma current $\mathrm{I}_{\mathrm{P}}$ of up to $1.4 \mathrm{MA}$, toroidal magnetic field $\mathrm{B}_{\mathrm{t}}$ of up to $3.1 \mathrm{~T}$ ) with high shaping capability. From 1996-2007, all plasma-facing components were converted from C to tungsten (W). Meanwhile, complete $\mathrm{W}$ coverage has been achieved; however, besides oxygen, carbon concentrations of about $1 \%$ are still observed in the plasma [20]. Besides substitution of the first-wall material, AUG was subjected to several further modifications, details of which are to be found in [29] and the references therein. The investigations reported here were all performed in the 2012/13 campaign in the divertor IId configuration, as shown in figure 1.

The versatile set of auxiliary heating and current drive systems comprises $20 \mathrm{MW}$ neutral beam injection (NI), up to $6 \mathrm{MW}$ ion cyclotron resonance heating (ICRH) coupled power, and $5 \mathrm{MW}$ electron cyclotron resonance heating (ECRH). Recently, in-vessel saddle coils capable of producing non-axisymmetric magnetic perturbations were installed. The set-up is composed of 16 B-coils which can produce a radial field with toroidal mode numbers of up to $\mathrm{n}=4$ [30]. The Discharge Control System (DCS) [31] is our tool for fully-automated and controlled execution of the plasma discharges. It supplies sophisticated real-time control methods comprising real-time diagnostic integration, dynamically adaptable multivariable feedback schemes, actuator management including load distribution schemes and a powerful monitoring and pulse supervision concept based on segment scheduling and exception handling. Proper plasma diagnosis is ensured by a broad range of diagnostic systems, several of them adapted to the real-time data processing and evaluation necessary for feedback control purposes. However, some scenarios used in this study required plasma shapes not ideal for diagnosing the plasma edge; and, in addition, pellet-induced perturbation can distort 
some measurements. Hence, several experiments presented here lack a full set of data, especially for characterization of the plasma edge. Most data shown in this paper were, therefore ELM-synchronized to improve the data quality. For some diagnostics prominently featuring in this study, more details are given here in brief. In the current configuration, the (thermo-) electric current into an outer divertor tile, measured as the voltage at a shunt resistor embedded in the tile mounting [32], can be employed as a simple and robust signal for monitoring an ELM. Function parameterization (FP) is used for identification of the plasma equilibrium. Scalar parameters such as the MHD stored energy, the plasma current or radial and vertical coordinates of particular geometric quantities are recovered in real time with a temporal resolution of typically $0.1 \mathrm{~ms}$ from magnetic data using parameterizations generated from an offline training procedure [33].

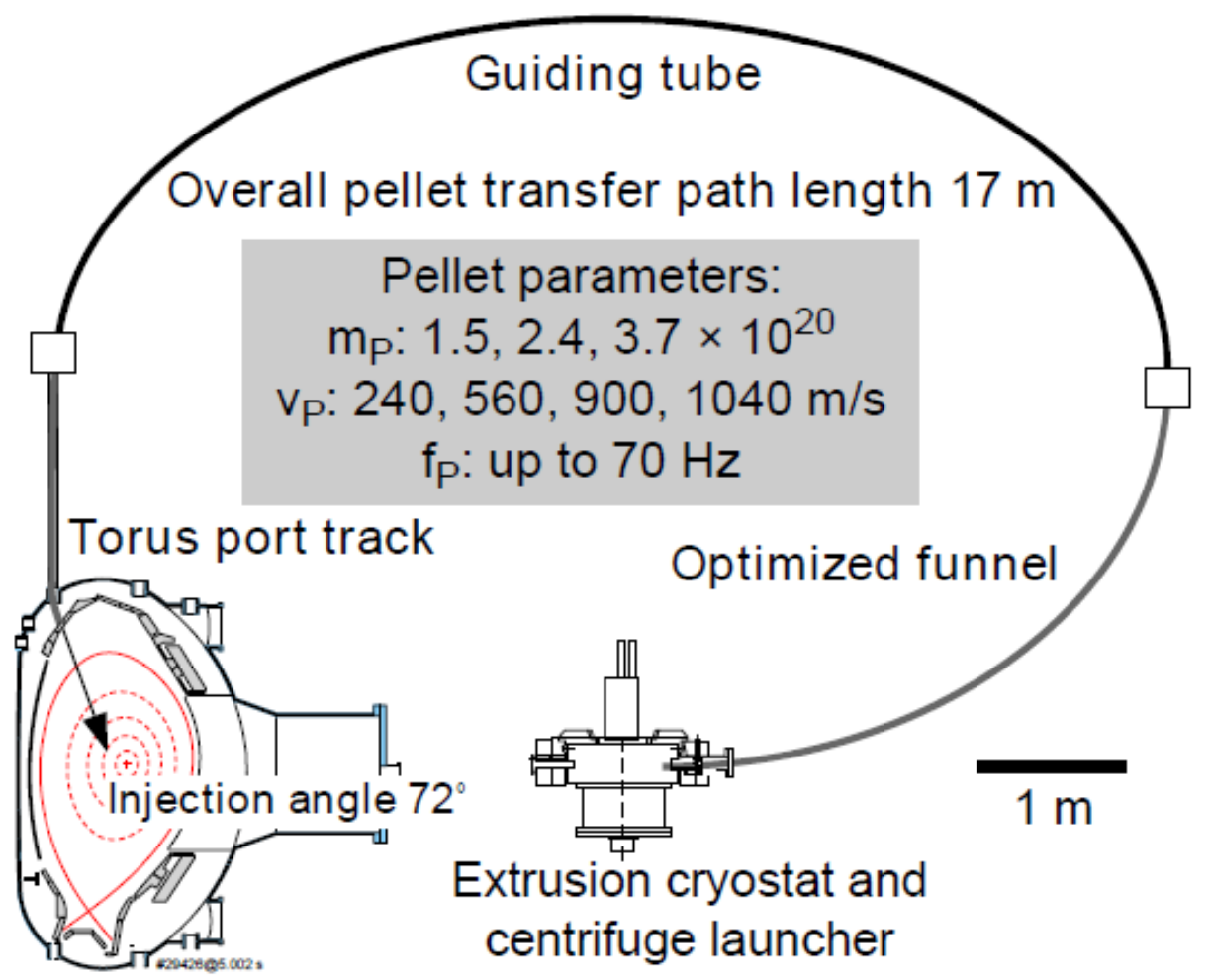

\section{ASDEX Upgrade with divertor IId}

Figure 1: Set-up as operated in the 2012/13 campaign. AUG with an all-W-coated wall, divertor IId and the full set of $B$ coils. The revitalized pellet launching system is now capable of covering a wide range of pellet parameters for both fuelling and ELM pacing applications.

Details of the enhanced ASDEX Upgrade pellet centrifuge launcher are reported elsewhere [34], only a brief description of its capabilities being given here. A sketch of the set-up is shown in figure 1, the high-speed inboard launching system is based on a centrifuge accelerator and a looping transfer system. Currently, the system is capable of reliably delivering pellets in standard operation with a particle content at the exit of the centrifuge in the range $m_{P}=1.5-3.7 \times 10^{20} \mathrm{D}$ in the velocity range $\mathrm{v}_{P}=240-1040 \mathrm{~m} / \mathrm{s}$ from the magnetic high-field side of the torus with repetition rates of up to $70 \mathrm{~Hz}$. (Even smaller pellets can be produced by applying "undersized" cutting, but at the expense of reduced reliability and enhanced size scatter). Within a given pellet train launched into a discharge both the pellet 
speed and size is fixed. However, repetition rates can be changed to a fixed fraction of the centrifuge revolution frequency.

The pellet observation system was recently upgraded and now includes two ultra-fast CMOS cameras. One of them, with a tangential view overlooking the whole poloidal cross-section of the pellet injection, was optimised for pellet tracking. The camera can be triggered for each pellet and captures a small region of interest which covers the whole pellet trajectory expected. This allows us to record fast framing movies of the ablating pellets with a frame rate between $150-200 \mathrm{kHz}$ in a distinct time window. During the movie post-processing the pellet position is calculated for each frame and stored in toroidal and flux coordinates as a function of time.

The performance usually achieved with the pellet system can be seen in figure 2, showing the result of a technical test dedicated to monitoring the reliability of pellet delivery and the size scatter of the arriving pellets. Pellets of the smallest possible standard size were launched at medium speed with a requested frequency of $10 \mathrm{~Hz}$. In order to allow optimal monitoring of mass deposition, a quiescent L-mode plasma was chosen to avoid disturbance by spontaneous and triggered ELMs. About $90 \%$ of the requested pellets arrive in the plasma; in turn, about $90 \%$ of the arriving pellets seem to be in good shape. Tattered pellets can easily be detected from their lack of ablation radiation recorded from a wide-angle-view diode which observes the entire ablation zone. The mass scatter of good pellets is about $\pm 10 \%$. Thanks to the precision of a stop cylinder-type centrifuge accelerator the speed scatter of less than $0.25 \%$ is negligible. The penetration depth of viable pellets also shows just a small scatter (pellets reach the flux surface $\left.\rho_{\text {pol. }}=0.7\right)$. For cases penetrating significantly less $\left(\rho_{\text {pol. }}<0.85\right)$ two pellet fragments of similar size were typically observed. Accordingly, the consistency of delivered viable pellets provides a sound basis for detailed investigations of ELM triggering.

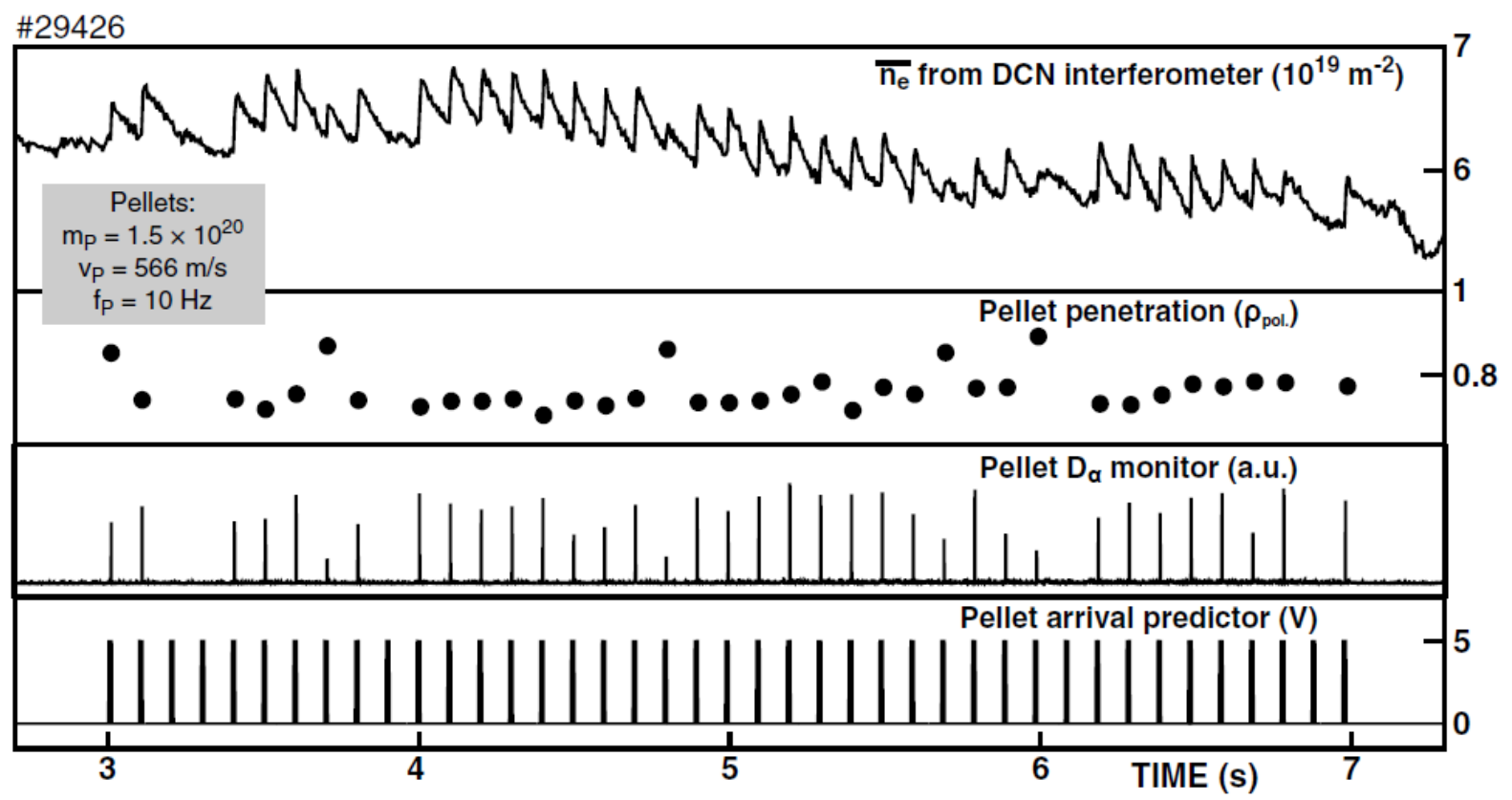

Figure 2: Pellet performance in a quiescent L-mode plasma. About $90 \%$ of the requested pellets arrive; about $90 \%$ of the arriving pellets show less than $\pm 10 \%$ mass and less than $0.25 \%$ speed scatter. 


\section{Investigations applying magnetic triggering}

With the pellet launcher still under renovation, first experiments resuming ELM pacing and triggering with the full-W wall were conducted, while using the technique of magnetic triggering. The technique relies on rapid vertical movement of the plasma column. Developed at TCV and mainly operated in the type-III ELM regime [22], it was ported to AUG and proved to work in the type-I regime as well [35]. It was further refined at JET and these socalled "kicks" are routinely applied for different purposes, e.g. maintaining stationary conditions at a heating power marginally above the L-H transition threshold by enforcing small regular ELMs [14].

In AUG with partial carbon wall coverage (AUG-C), reliable and reproducible locking of the ELMs to the applied vertical "wobbling" motion was achieved well within the operational range of the plasma position and shape control system in the frequency range of $30-70 \mathrm{~Hz}$. Since then, the control system has been upgraded by replacing the previous transputer-based control system with the DCS, which decreased its response time to about 2 ms. However, the plasma response time is mainly limited by the vertical field coils. Recalling the scenario known to deliver the best plasma response with respect to the achieved vertical motion and ELM locking made us choose operation with a driving frequency of $55.6 \mathrm{~Hz}$ (18 ms cycle time).

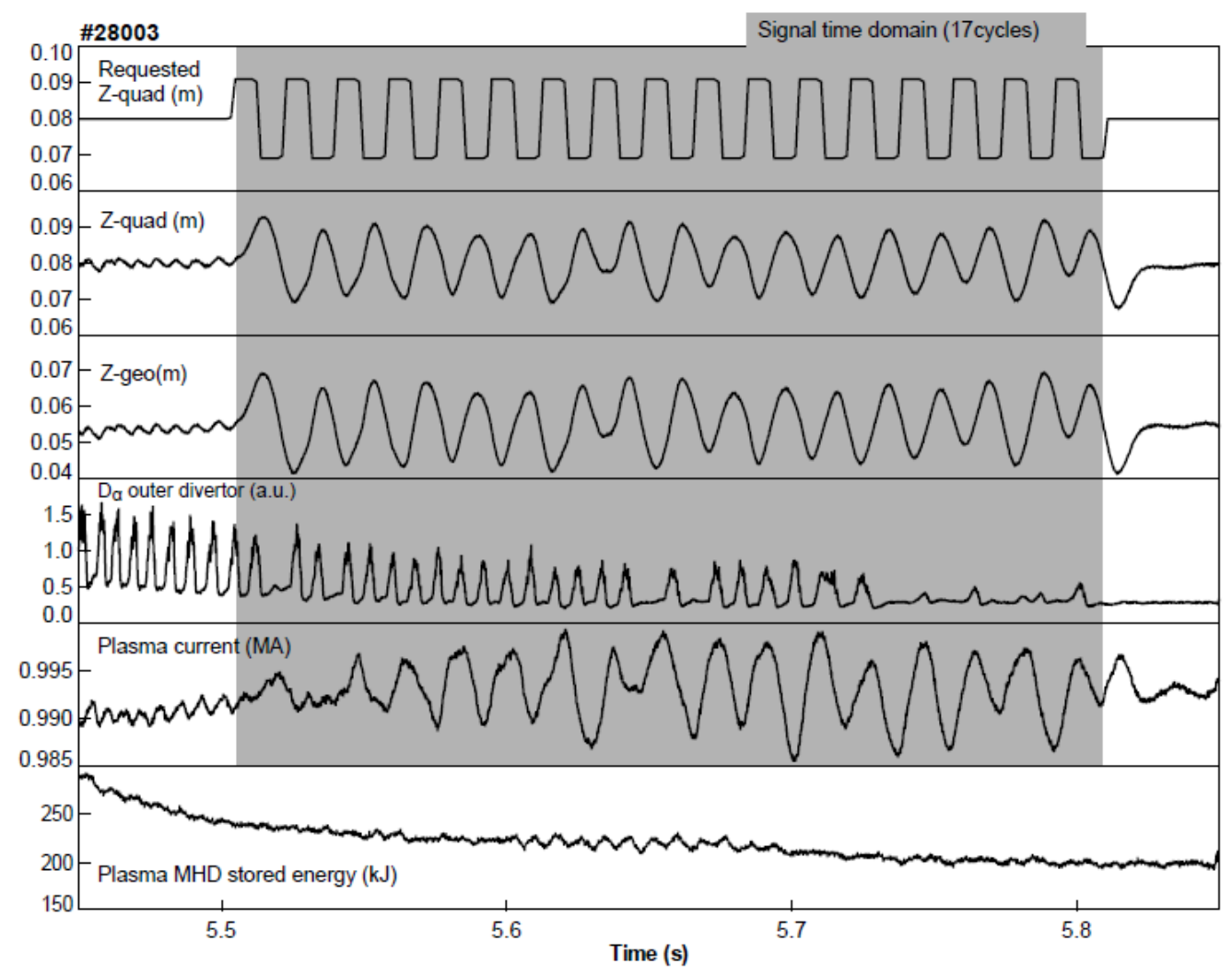

Figure 3: Attempt to recover ELM frequency locking by fast vertical motion of the plasma column ("magnetic triggering"). No indication of locking is found for a plasma scenario that showed perfect locking in AUG-C. The time range selected for generating the averaged data shown in figure 4 is shaded. 
Several attempts to apply the method in order to avoid ELM-free phases just after entering the $\mathrm{H}$-mode did not show the desired response, no impact of the motion on the ELM behaviour being detected. Consequently, an approach was applied in order to check the effectiveness of the technique by retrying the scenario from the successful experiment reported in [35]. A configuration showing stable and robust operation with operational parameters $\left(\mathrm{I}_{\mathrm{P}}=1.0 \mathrm{MA}\right.$; $\mathrm{B}_{\mathrm{t}}=2.5 \mathrm{~T}$; edge safety factor $\mathrm{q}_{95}=4.8$; plasma elongation $\kappa=1.79$; upper and lower triangularity $\delta^{\mathrm{u}}=0.129$ and $\delta^{\mathrm{l}}=0.395$, respectively; auxiliary heating power by neutral beam injection $\mathrm{P}_{\mathrm{NI}}=5 \mathrm{MW}$ ) very close to those in the recent scenario was selected. In contrast to the zero gas fuelling, this time a steady gas puff of the order of $4 \times 10^{21} \mathrm{D} / \mathrm{s}$ had to be applied to avoid impurity accumulation, causing a higher ELM frequency as well. Once again, no response could be observed with respect to the applied motion. In order to provide, at least temporarily, optimum locking conditions, a scan of the ELM frequency downwards toward the driving frequency of $55.6 \mathrm{~Hz}$ was made. Prior to the wobbling phase the heating power and gas rate were reduced, resulting in a gradual decrease of the plasma energy and the ELM frequency. Once again, as shown in figure 3, there is no indication of ELM frequency locking. The inability to achieve a significant impact on the ELM behaviour is observed despite large vertical peak-to-peak wobbling amplitudes of about $20 \mathrm{~mm}$. This resulted in a maximum speed greater than $3 \mathrm{~m} / \mathrm{s}$. For AUG-C, locking was already achieved for wobbling amplitudes of about 5-7 mm, which corresponds to a maximum speed of $1 \mathrm{~m} / \mathrm{s}$. For parameters as applied in the recent experiments at AUG-W, ELM frequency locking already set in at the first harmonic at AUG-C. For the sake of machine security, no higher wobbling amplitudes were applied this time.
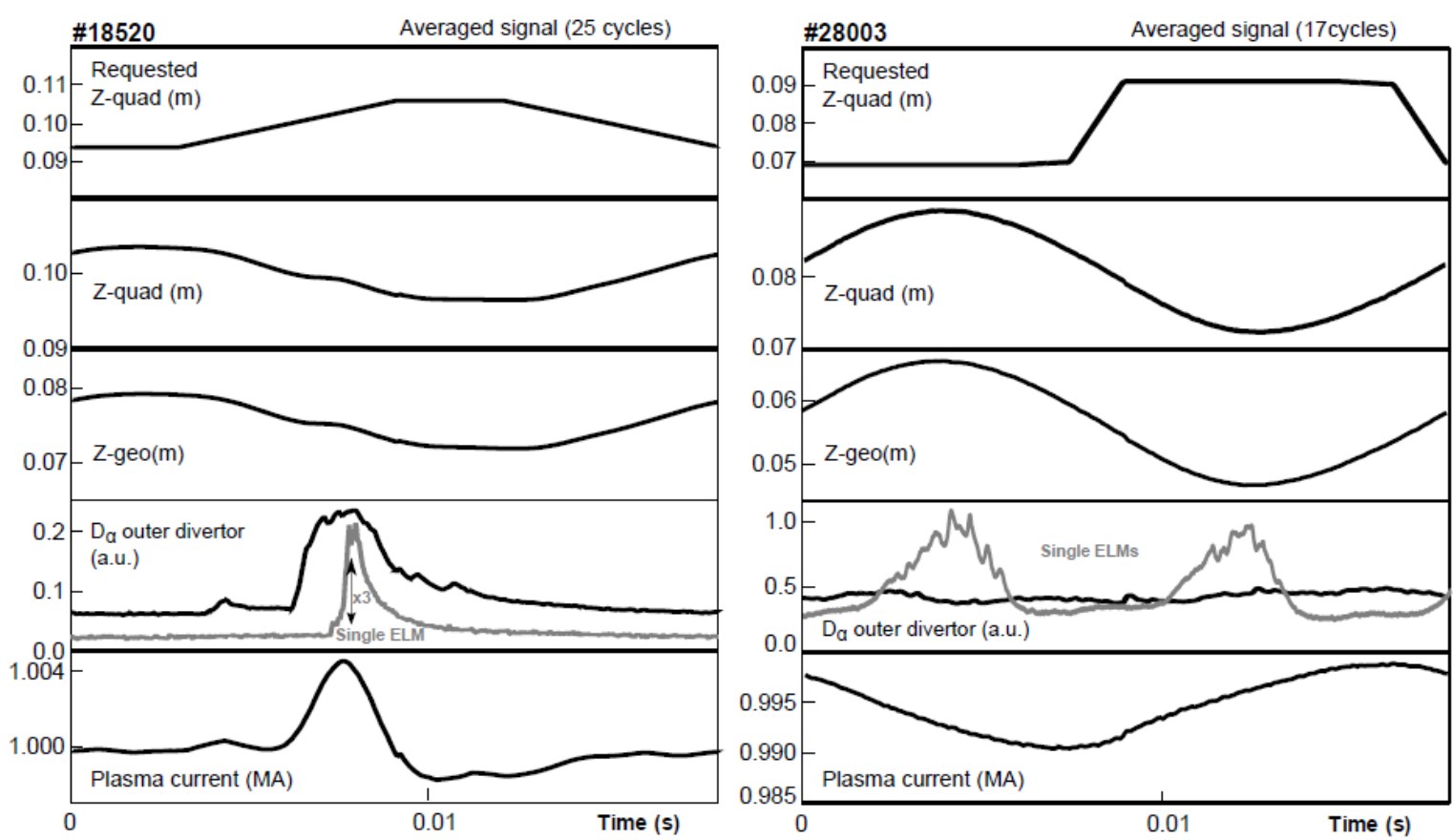

Figure 4: Comparison of ELM response to vertical plasma movements between AUG-C (left) and $A U G-W$ (right). Averaging over several motion cycles shows a clear response in the $A U G-C$, whereas no response appears in the AUG-W case even though stronger wobbling (note different scales) is applied. Note also differences in the ELM dynamics (single ELM traces in grey). 
The drastic difference in ELM response to the wobbling becomes clear when signals over several motion cycles are compared. Figure 4 displays cases for AUG-C (left) and AUG-W (right). Data representing the AUG-C case are obtained by taking the 25 wobbling cycles from the discharge shown in figure 1 of [35], while the AUG-W counterpart is obtained by averaging over the 17 cycles indicated by the shaded area of figure 3. Both discharges are very similar, apart from the additional gas fuelling required in the AUG-W case, in which the motion was also much more pronounced. Clear locking of the ELMs to the wobbling frequency is achieved for the AUG-C case since both frequencies are identical, although a slight timing jitter of a few ms appears. The ELM triggering probability is highest when the downward speed reaches its maximum. A clear impact of the ELM on the plasma motion and the plasma current is also observed. In contrast, there is no indication of locking in the AUGW case. Notably, there is already a significant difference in the ELM dynamics (monitored by $\mathrm{D}_{\alpha}$ radiation from the outer divertor strike point) of the two cases. For the AUG-C case, ELMs (both locked and spontaneous) are stronger and shorter than in the AUG-W case. For comparison, also the dynamics of single ELMs is shown (solid grey lines). With the requested and observed motion in antiphase, the plasma position control system is at its limit.

\section{Deriving the ELM trigger lag time and investigating the impact of pellet parameters}

With the pellet system available, the first task was to ascertain a trigger lag time as observed in JET with the ILW (JET-ILW) in AUG-W as well and investigate if the magnitude of the pellet-imposed local perturbations has an impact. The same target plasma scenario was chosen as for the magnetic triggering experiments, since it showed sufficiently low but stable spontaneous type-I ELMs and was also reasonably robust against the additional fuelling inevitably coming with the pellets. With a gas puffing rate of about $7 \times 10^{21} \mathrm{D} / \mathrm{s}$, it typically developed a spontaneous initial ELM frequency $\mathrm{f}^{0}{ }_{\text {ELM }}$ of about $50 \mathrm{~Hz}$. As every pellet has a significant impact on the plasma under the conditions at ASDEX Upgrade, a sufficiently low pellet rate has to be chosen for a probing experiment. The temporary perturbation by the pellets, also resulting in a transient increase of the ELM rate, was fully recovered after $100 \mathrm{~ms}$. A pellet rate of $10 \mathrm{~Hz}$ was therefore applied. With this rate the ELM appearance still proved to be uncorrelated to the pellets' arrival. Thus, every pellet hits virtually the same plasma at an arbitrary time point in the spontaneous ELM cycle. ELMs and pellets do show a significant impact on many plasma parameters allowing any possible effect of pellet ablation on the ELM evolution to be monitored. Thus, in almost all cases it can definitely be ascertained if a pellet triggers an ELM or not. 


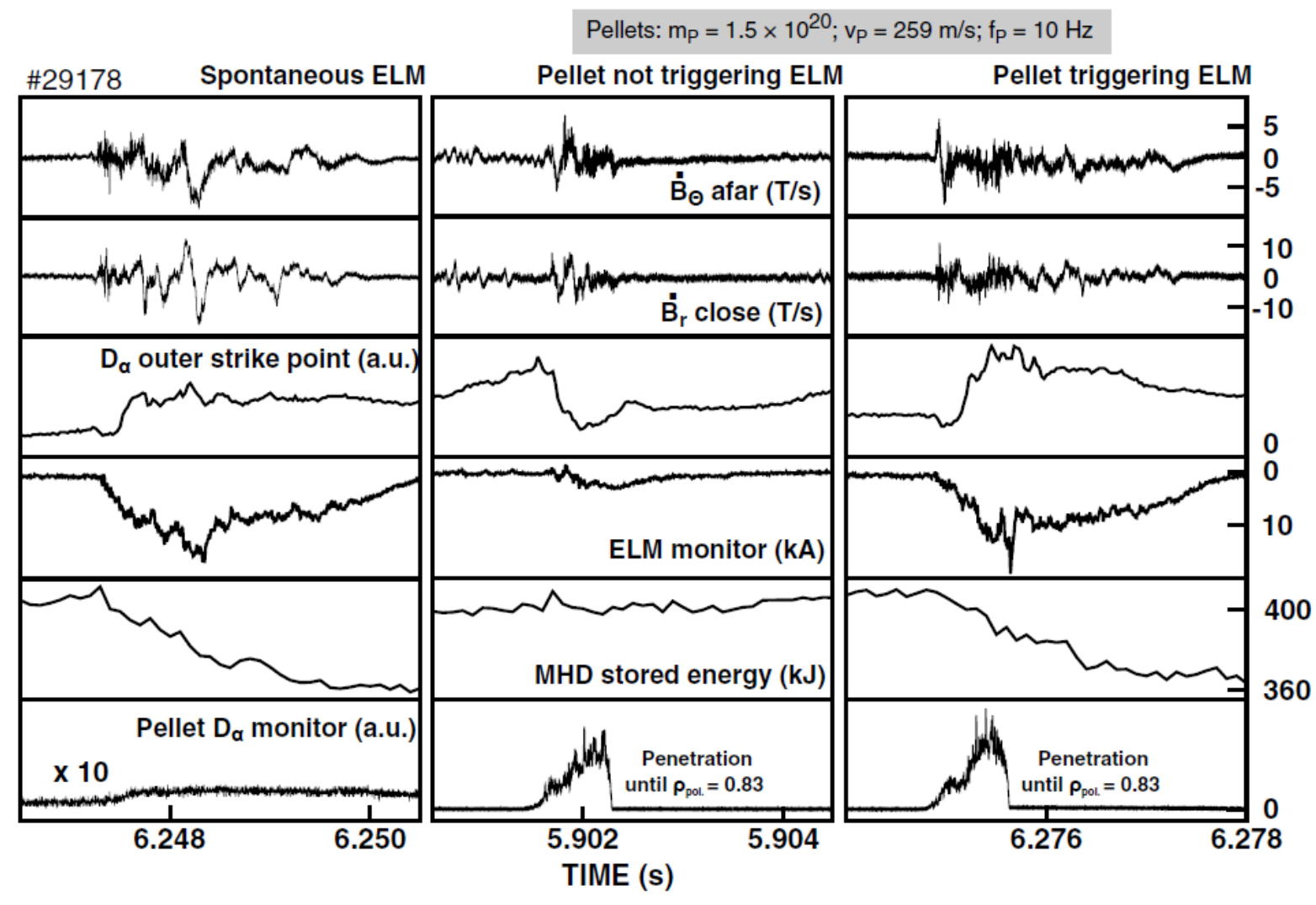

Figure 5: To distinguish between pellets triggering an ELM and those not resulting in direct triggering, several monitor signals can be used. A triggering pellet (right) combines the essential features of both a spontaneous ELM (left) and a mere pellet event (middle). The pellet impact on the plasma energy proved to be a most convenient monitor. It was therefore primarily used.

To distinguish between pellets triggering an ELM and those not resulting in direct triggering, several monitor signals can be used. An example is shown in figure 5, displaying data recorded for a spontaneous ELM (left), a mere pellet not resulting in direct triggering of an ELM (middle) and a pellet directly triggering an ELM (right). The pellet ablation monitor, plotted in the bottom row, and the measured pellet penetration show that the two pellets involved are virtually identical. Although the ELM-induced radiation at the plasma edge causes some weak response in the pellet monitor signal as well, the pellet ablation onset can clearly be determined. Our previous investigations showed both pellets and ELMs create strong and characteristic MHD activity [36]. To determine the magnitude of the MHD perturbation, we made measurements of $\frac{d B_{\theta}}{d t} \equiv \dot{B}_{\theta}$ and $\frac{d B_{r}}{d t} \equiv \dot{B}_{r}$ with Mirnov coils mounted inside the vessel. Both signals show the expected response to pellet and ELM-created perturbations, the pellet perturbation being significantly shorter than the ELM response. Closer analysis of the magnetic perturbation spectra and toroidal mode number based on wavelets revealed both perturbations to have distinct frequencies and toroidal mode numbers [36, 37]. For the pellet triggering the ELM, the observed correlating MHD activity is the super-positioning of an initial pure pellet perturbation followed by an ELM perturbation. Also, the $D_{\alpha}$ radiation from the region around the outer divertor strike point and the ELM monitor signal derived from the measured outer divertor current show a specific response to a pellet or an ELM, the two components being observed only in the case of a triggered ELM. 
The most convenient way to identify if a pellet triggers is by analysing its impact on the plasma-stored MHD energy $\mathrm{W}_{\text {MHD }}$. Results obtained by applying different methods match very well. Since this method seemed most adapted to proper visualization, it was applied for all analysis. For the timing in the ELM cycle, we took the time elapsed since the previous ELM ( $t=0$ at the onset of the sharp drop in stored energy) at the onset of pellet ablation (measured by the diode).

The temporal evolution of $\mathrm{W}_{\mathrm{MHD}}$ and the pellet ablation for a reference spontaneous ELM and the three characteristic cases observed are shown in figure 6 , all taken from a single discharge with pellets of the smallest mass (in standard operation) and slowest speed available. The reference spontaneous ELM (uppermost box) shows the typical ELM-induced loss of MHD stored energy $\Delta \mathrm{W}_{\mathrm{MHD}}$ to be about $13 \%$ of the stored energy before the ELM, $W_{0}$. An unperturbed ELM cycle lasts about 25 ms. Response to virtually identical pellets (comparable ablation monitor traces and penetration depth) arriving at a different elapsed time since the previous ELM crucially changes. No significant immediate response is found with the pellet arriving early in the ELM cycle (case (A)), and no prompt ELM is triggered by the pellet perturbation created in the pedestal during ablation. The pellet arriving a few ms later in the cycle (case (B)) clearly triggers an ELM, but the size measured by the loss of energy normalized to the stored energy before the ELM, $\Delta \mathrm{W}_{\mathrm{MHD}} / \mathrm{W}_{0}$, is reduced with respect to a full-sized spontaneous ELM. The pellet arriving around $10 \mathrm{~ms}$ after the previous event triggers a full-sized ELM. Notably, the full-sized ELM is triggered at a time when the appearance of a spontaneous ELM is still very unlikely. The impact of the pellets on the ELM cycle duration due to the fuelling impact also becomes clear from figure 6. Due to the pellet fuelling a few ELMs following after pellet injection appear at an enhanced rate. Fuellinginduced ELMs are easily discriminated from promptly triggered ones and are not the subject of this investigation. Notably, still as in AUG-C prompt ELMs are triggered very soon (typically in less than $0.1 \mathrm{~ms}$ ) after the onset of pellet ablation. Thus, as shown in a dedicated earlier study [38], the pellet-imposed perturbation finally causing (or growing into) the ELM is located within the pedestal.

Normalized energy losses for pellet-triggered ELMs were derived as indicated in the lower box of figure 6; for cases with no pronounced pellet-induced energy drop (can be identified by $\mathrm{dW}_{\mathrm{MHD}} / \mathrm{dt}>0$ at the end of pellet ablation) as in case (A) the difference in $\mathrm{W}_{\mathrm{MHD}}$ between the onset and end of pellet ablation is taken. 


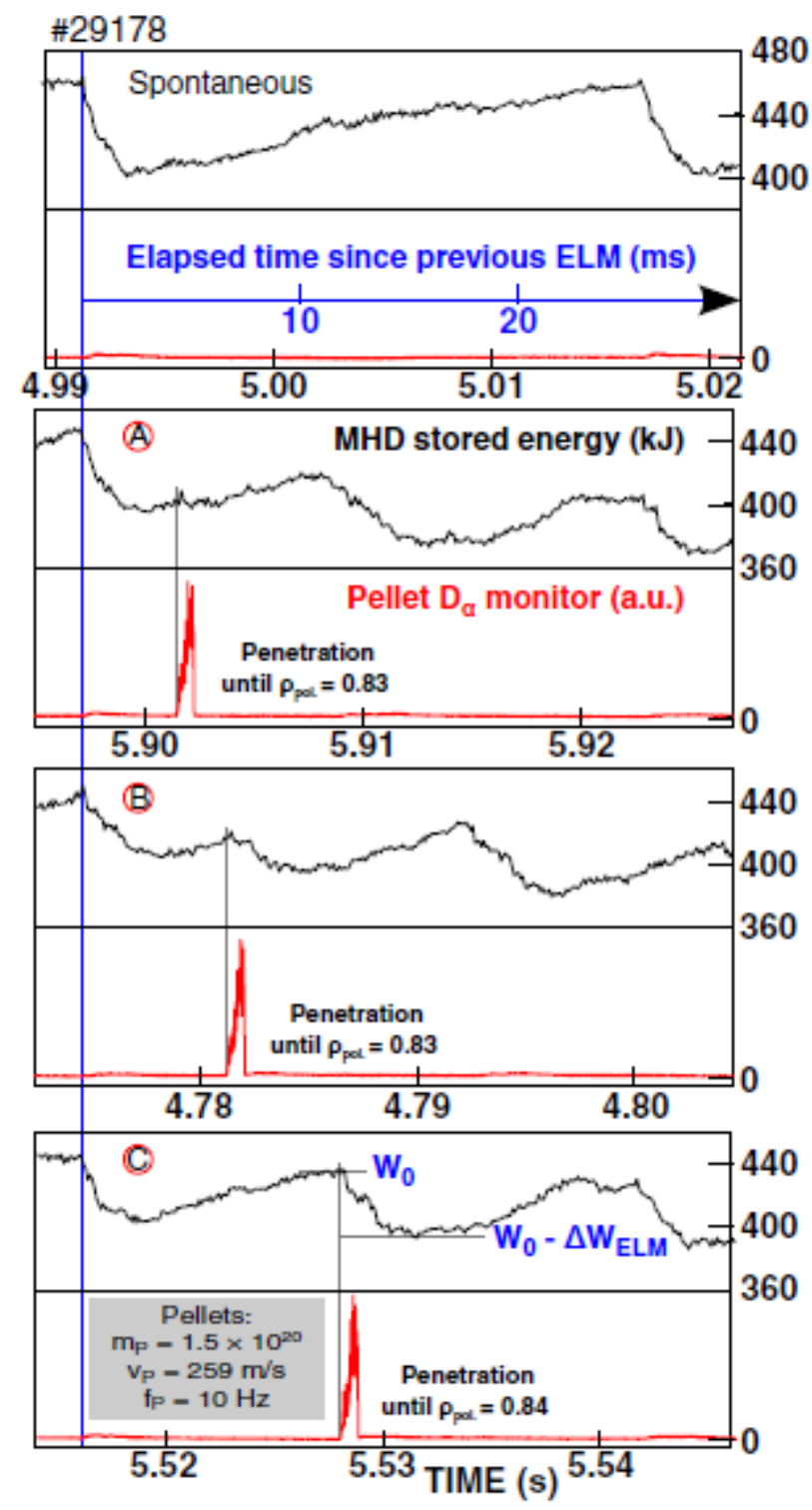

Figure 6: Pellets probing their ELM triggering capability at different times within the ELM cycle. The plasma energy loss induced by the pellet proved to be a useful monitor. Early in the cycle no ELM is triggered (A), later ELMs are triggered first smaller (B) and finally (C) as large as the spontaneous reference ELM (uppermost box).

To correlate the observed behaviour with the recovery of the edge after an ELM crash, data from 90 mostly unperturbed ELMs were used to show the evolution of the electron pressure, temperature and density at the pedestal top in figure 7. To increase the data quality, ELMcoherent averaging was used. The typical pedestal recovery phases described in detail in [39] can be observed. While the temperature recovers in three main phases (2ms fast recovery, $3 \mathrm{~ms}$ constant phase and 10ms final recovery), the density recovers in 3ms. Unlike in previous work, the density pedestal recovers during the first phase of the temperature recovery.

In order to investigate a possible influence of the pellet imposed local perturbation on the potential to trigger an ELM, the pellet ablation rate was varied by changing the pellet speed and mass. Data were collected from many virtually identical discharges. Covering the entire accessible range for standard operation, pellet parameters were varied, being referred to by colour (speed) and symbol (mass). They are all displayed in the upper part of figure 8, 
plotting the loss of plasma energy normalized to plasma stored energy before the ELM, $\Delta \mathrm{W}_{\mathrm{ELM}} / \mathrm{W}_{0}$, versus the elapsed time since the last ELM. Cases shown in figure 6 ((A) to (C) and $\mathrm{S}$ for the spontaneous reference case) are indicated. For reference, also data for spontaneous ELMs from several reference phases are shown and, in the lower part, the $\mathrm{W}_{\mathrm{MHD}}$ evolution in a spontaneous ELM cycle (as obtained by ELM-coherent averaging over an ensemble taken from several discharges). Here, the distinct phases observed during pedestal recovery are also indicated.

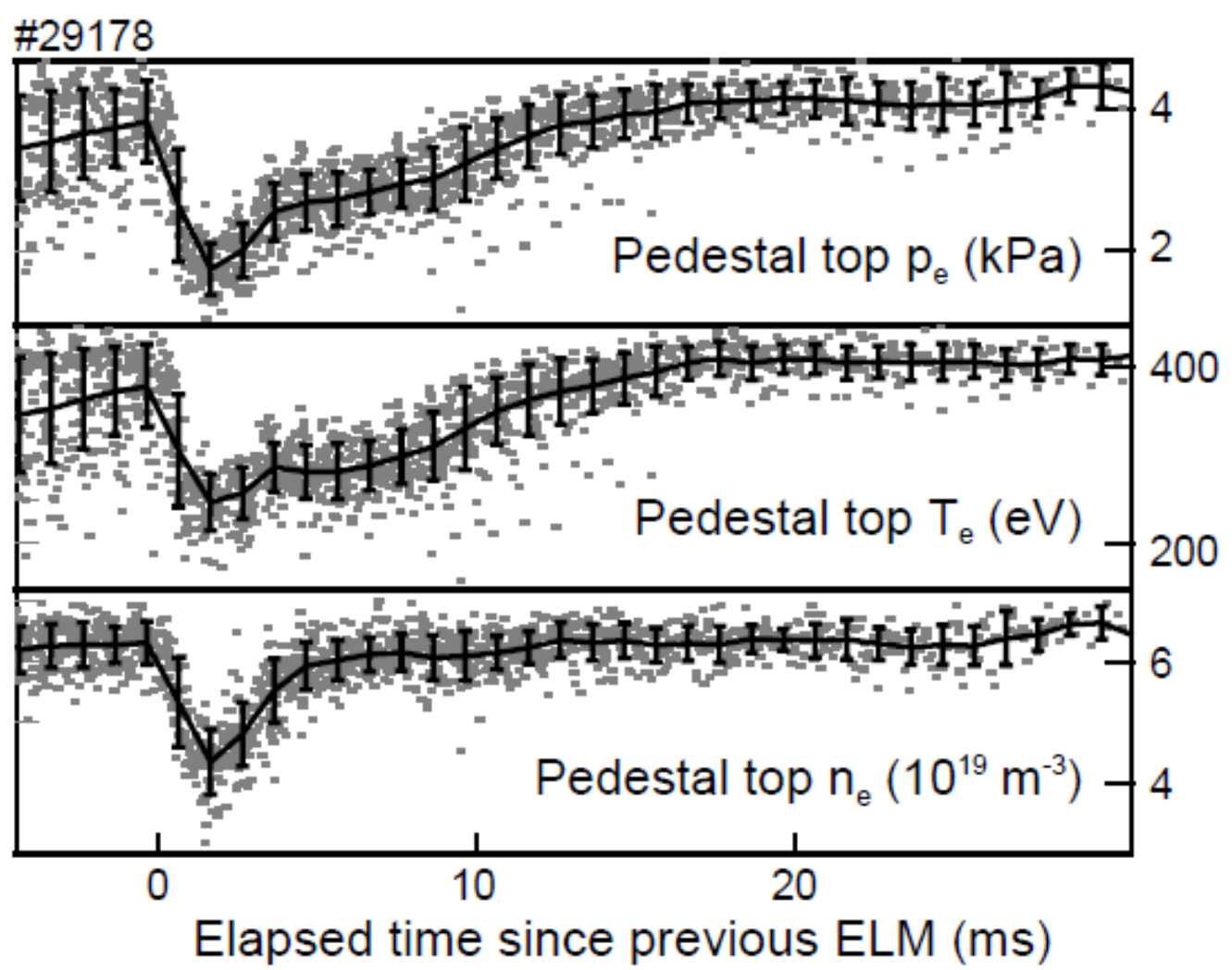

Figure 7: Evolution of density, temperature and pressure at the pedestal top during a spontaneous unperturbed ELM; data obtained by ELM-coherent averaging over single events from the pre-pellet reference phase.

In order to differentiate reliably the observed response between ELM triggering and nontriggering pellets, the analysis tool was applied to a reference ELM. This reference ELM was generated by data averaging over many ELMs occurring in this scenario. The response was modelled on the assumption that a pellet arriving at any time has no impact on $\mathrm{W}_{\mathrm{MHD}}$, i.e. no additional ELM is triggered. It shows (solid grey curve in figure 8) a positive response in cases where a pellet is injected while the previous spontaneous ELM is still on-going, dropping to slightly negative values afterwards. This expected nil response agrees well with the data recorded up to about $7 \mathrm{~ms}$, the scatter within the data set being taken into account. A clear transition starts at about $7 \mathrm{~ms}$, terminating the agreement of the data with the modelled no-triggering response, and hence this phase with zero trigger probability ends. Finally, at about $10 \mathrm{~ms}$, triggered ELMs reach the full magnitude of a spontaneous counterpart and reliable triggering is observed. Within the transition phase lasting from about 7 to $10 \mathrm{~ms}$, ELMs are triggered, but their corresponding energy losses show a reduced size. After $10 \mathrm{ms,}$ no change in size of triggered ELMs is observed despite the fact that the pedestal stored energy further recovers. For this plasma scenario, this crossover from a stable edge to 
conditions where at least a strong local perturbation can trigger an ELM correlates well with the phase where the fast recovery of the edge temperature gradient comes to an end. Notably, most spontaneous ELMs occur between 20 and 35 ms, but some already take place shortly after $10 \mathrm{~ms}$, at a time when triggering becomes achievable. No spontaneous ELM appeared between 12 and $20 \mathrm{~ms}$.

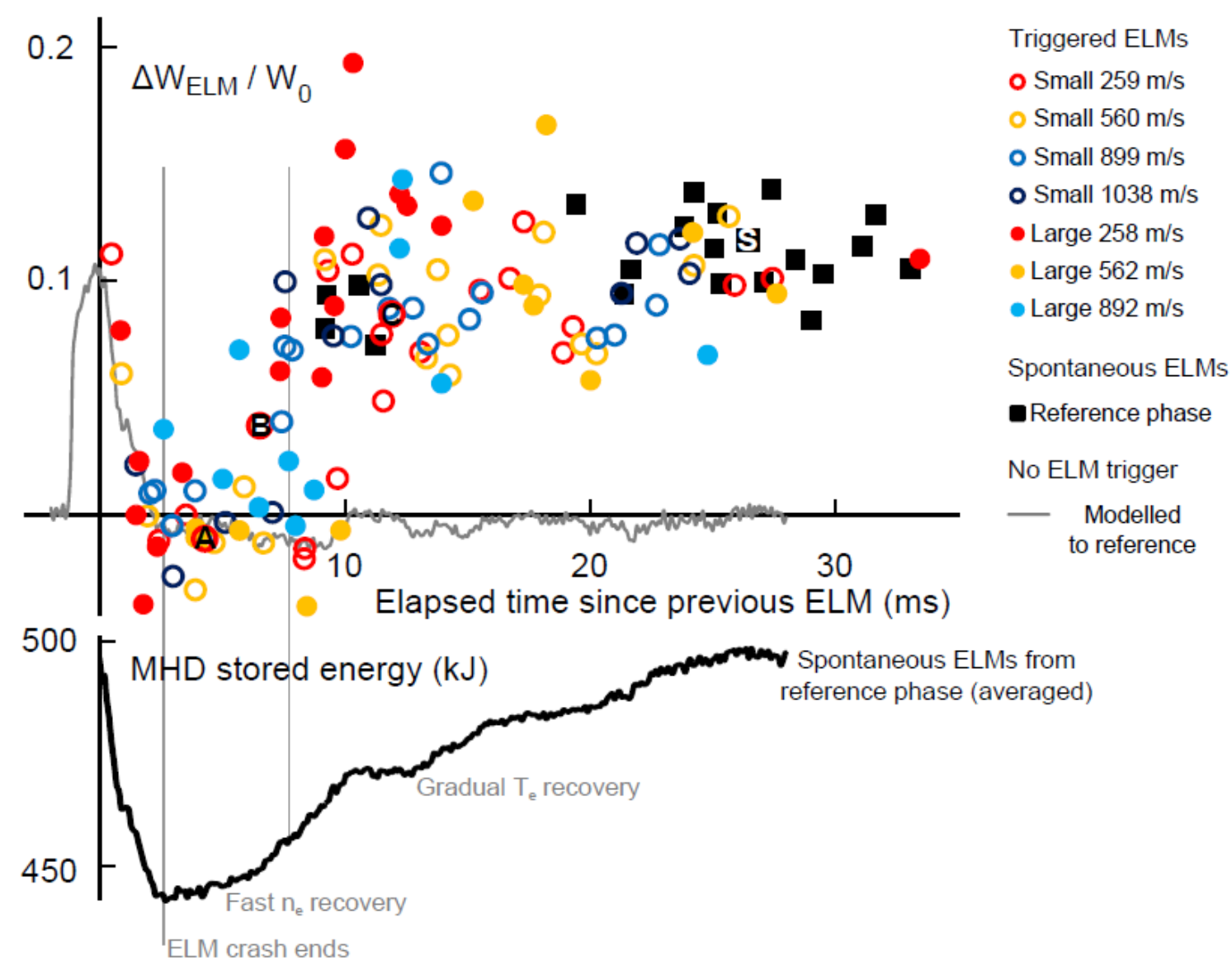

Figure 8: Top: Loss of plasma energy normalized to plasma stored energy before ELM versus time elapsed since onset of the previous ELM. Data obtained for different pellets and from spontaneous ELMs. The modelled response of a pellet causing no impact on the ELM cycle is displayed as a solid grey line. Bottom: Averaged plasma energy evolution during a spontaneous ELM cycle.

No indication is found that the magnitude of the pellet perturbation in the parameter range covered plays a role for the resulting trigger lag time. Evidently, large pellets can cause stronger ELM-induced losses since they enhance convective losses by creating a massive particle flux through the edge, but they do not allow earlier triggering. Hence, a variation of the local pellet-imposed perturbation in the pedestal ranging from the smallest (fast, small pellets) to largest (slow, large pellets) achievable has no impact on the pellet trigger potential. It is worth mentioning that the pellet tracking system confirmed that all pellets penetrate well beyond the pedestal top (small, slow ones penetrating to at least $\rho_{\text {pol }}=0.9$, the pedestal top being situated at $\rho_{\mathrm{pol}}=0.97$ at most). 


\section{Attempts for pacing and mitigation}

With confirmation of the appearance of a lag time for pellet ELM triggering, this being moreover independent of the pellet parameters, an attempt was made to resume pacing in a suitable scenario using the smallest standard pellet size. This involved slightly adapting the scenario hitherto used, since applying higher pellet rates would otherwise have caused a change too drastic of the target plasma. Adding an additional NBI beam increasing $\mathrm{P}_{\mathrm{NI}}$ from 5.0 MW to 7.5 MW (raising the normalized plasma pressure from about 1.6 to about 1.75) was sufficient, while still maintaining an acceptable $\mathrm{f}_{\text {ELM. }}^{0}$. A first approach using a pellet frequency $f_{P}=50 \mathrm{~Hz}$, a rate found most appropriate to achieve full pacing in AUG-C [16], showed just modest success. Some sequences could be established where the ELM frequency was locked to $\mathrm{f}_{\mathrm{p}}$. However, due to the fuelling side-effect of the pellets, the spontaneous ELM frequency was raised too much and persistent pacing could not be achieved. System enhancements allowing a $70 \mathrm{~Hz}$ pellet rate were required to finally achieve persistent pacing. Figure 9 shows an example where clearly $\mathrm{f}_{\mathrm{P}}=\mathrm{f}_{\mathrm{ELM}}=70 \mathrm{~Hz}$ is established as required for full pacing. However, taking a closer look at the timing of the ELM onsets reveals that some ELMs are not promptly triggered by the pellets but obviously appear spontaneously shortly before pellet arrival. This is attributed to the fuelling impact of the pellets, increasing the spontaneous ELM frequency. Since all these pellets arrived just after a spontaneous ELM within the trigger lag time, no additional ELMs are triggered and the requested frequency is precisely maintained. Hence, it seems that full pacing was only marginally achieved. In AUG$\mathrm{W}$ the ultimate potential of the pellet system had to be applied in order to achieve pacing under marginal conditions while in AUG-C it could be established hassle-free. This shows strong evidence of ELM control in an all-metal-wall tokamak becoming more intricate.

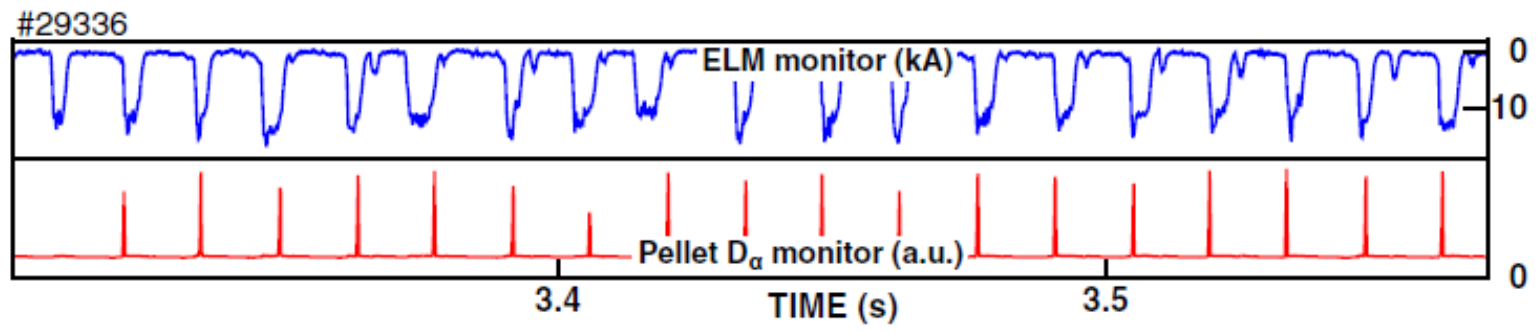

Figure 9: Reliable and persistent ELM pacing is resumed at $f_{P}=f_{E L M}=70 \mathrm{~Hz}$ in AUG-W; however, not all ELMs are promptly triggered since pellets alter the spontaneous ELM rate by their fuelling as well.

Notably, small pellets applied in this pacing approach result in modest but distinct density enhancement without any loss of energy. This already indicates favourable particle fuelling behaviour, as discussed later in this paper.

Beyond the question under what conditions mere ELM pacing can be achieved, it is essentially the potential for ELM mitigation that matters. Indications of mitigation becoming more intricate also merge from the latest investigations performed at JET-ILW. There, pellet ELM pacing resulted in enhancement of $\mathrm{f}_{\mathrm{ELM}}^{0}$ by a factor of about two, but only minor ELM mitigation with respect to $\Delta \mathrm{W}_{\mathrm{ELM}} / \mathrm{W}_{0}$ and virtually no reduction of even more crucial peak heat flux could be achieved [25]. For our pacing experiment, we made essentially the same observation. The effect pacing has on the ELM size in terms of $\Delta \mathrm{W}_{\mathrm{ELM}} / \mathrm{W}_{0}$ can be developed from the sequences displayed in figure 10. An ensemble of ELMs each is taken for spontaneous ELMs from the reference phase and from the pacing phase reached during the pacing approach, as highlighted in yellow. While there is sound enhancement of the ELM frequency by a factor of 1.6, no corresponding mitigation of ELM-induced energy losses takes 
place. Comparing the two ensembles, one has a weak mitigation effect but only by a factor of about 1.2. Since there is no difference of plasma energy in the two phases, as a consequence at least inter ELM losses must be reduced by the pacing.

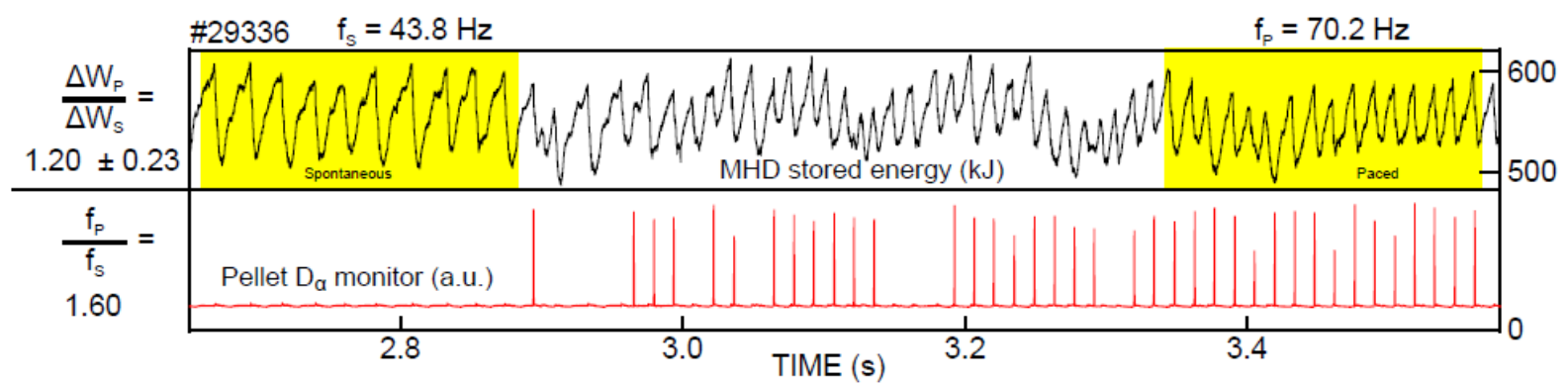

Figure 10: While pacing increases the ELM frequency by about 1.6 times, ELM-induced plasma energy losses are not reduced accordingly. Ensembles taken for the analysis are highlighted in yellow.

To allow measurement of the heat flux to the strike points using infrared thermometry, a slight change of the plasma positioning became necessary. This made the scenario more prone to (pellet) fuelling-induced reduction of energy confinement, resulting in a modest decrease of $\mathrm{W}_{\text {MHD }}$ during the pellet pacing phase. A modest reduction of the peak heat flux during ELMs was observed during the pacing phase with respect to the reference phase, but only in proportion to the plasma energy reduction. Therefore, no observable net reduction of heat flux took place.

With respect to the importance of the ELM mitigation issue to ITER, further efforts are required to find out the reason for the strongly different finding of these AUG-W and the recent JET-ILW results in respect of early results from AUG-C [16] and very recent ones from DIII-D reporting adequate mitigation in a regime with strong (12 times) enhancement of $\mathrm{f}_{\mathrm{ELM}}$ [40]. Of particular interest could be an approach to enhance significantly $\mathrm{f}_{\mathrm{ELM}}$ (by a factor of 10 rather than $1.6-2$ ) in an all-metal-wall tokamak. This can hardly be achieved at AUG with the pellet particle content inevitably significant with respect to the plasma particle inventory causing a significant fuelling side-effect, combined with quite high spontaneous ELM rates in a mid-size tokamak. Since it is mainly designed to handle this task, appropriate investigation had to wait for the JET HFPI system to be revised to full design performance as expected for early 2015.

\section{Trigger lag times in different scenarios}

An obvious question arising from the observation of a trigger lag time in AUG-W is: what are the underlying reasons why under certain circumstances a pellet can trigger an ELM while it cannot for others? As a first step we conducted experiments to determine trigger lag times for a few different plasma conditions with different separatrix power flux, causing changes of the ELM recovery dynamics. To begin with, the plasma scenario used for the pacing demonstration was taken. From the pacing experiment it can already be concluded that there must be a lag time, since the pellets injected just after an early spontaneous ELM did not trigger. Since pacing could be achieved at $70 \mathrm{~Hz}$, the lag time has to be shorter than $1 / 70 \mathrm{~Hz}=14.3 \mathrm{~ms}$. Notably, exercising the perturbative trigger probe technique, applying $\mathrm{f}_{\mathrm{P}}=10 \mathrm{~Hz}$, seems to allow a better temporal resolution of the lag time, as can be concluded 
from plot (A) in figure 11. The plot displays data obtained from reference phases for spontaneous ELMs, for a few paced ELMs and for triggering probe pellets injected at $10 \mathrm{~Hz}$. The data set shows consistent behaviour for the evolution of achievable ELM size along an ELM cycle; the trigger lag time is about $8-10 \mathrm{~ms}$.
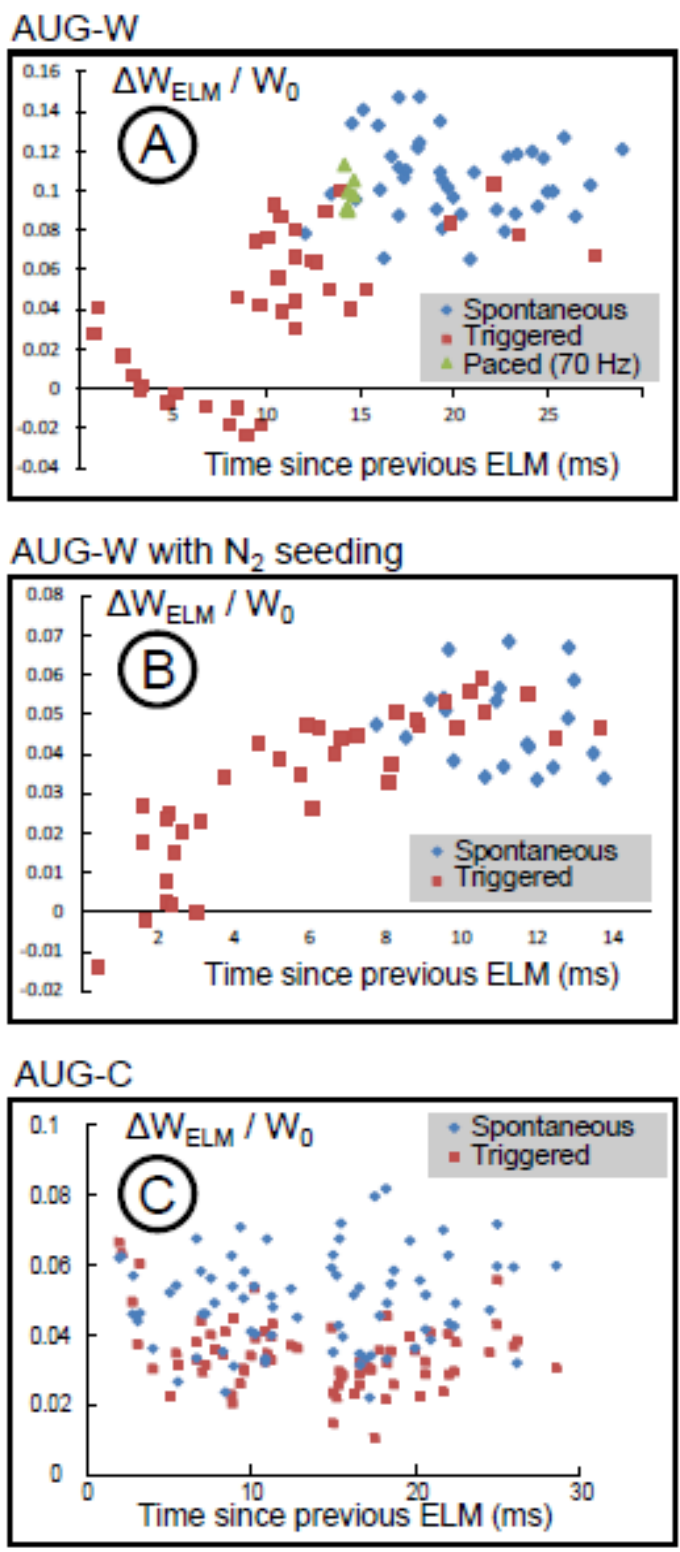

Figure 11: Loss of plasma energy normalized to plasma stored energy before ELM versus time elapsing since the onset of the previous ELM for different configurations. (A) In the pacing demonstration scenario, spontaneous ELM cycles last between 13 and 30 ms. Pacing results in more precise ELM timing, but does not notably alter ELM losses. Probe triggering at $10 \mathrm{~Hz}$ reveals a trigger lag time of about $8-10 \mathrm{~ms}$. (B) Operating at higher heating power (requiring additional $N_{2}$ seeding) shortens the lag time to about 2- $3 \mathrm{~ms}$. (C) In AUG-C, no indication of the lag time was observed [38].

Some exploratory shots were made at the end of the campaign to find out if trigger lag times can also show significantly different values. Two scenarios with rather different auxiliary 
heating powers were selected. The first aimed at demonstrating the ITER baseline scenario on ASDEX Upgrade [41], it being characterized by a highly triangular shape and significant core heating to avoid $\mathrm{W}$ accumulation. The operational space for this scenario was found to be rather restricted, particularly by the need to keep $\mathrm{f}_{\mathrm{ELM}}$ sufficiently high by ensuring effective flushing of impurities. To prevent an intolerably low ELM rate, pellet pacing as a tool for ELM frequency sustainment as successfully applied in AUG-C [42] was requested. Scenario stabilization attempts by applying pellets to keep a minimum ELM rate showed only slight improvements, resulting essentially from enhanced pellet particle fuelling efficiency as compared with gas puffing. Pellets were found to show very low trigger probability, especially during phases in which triggering became most desirable when spontaneous ELM cycles became extremely long and impurity accumulation set in. Under these circumstances, the longest lag times hitherto were also observed. One case is shown in the left part of figure 12. After the ELM crash, the pedestal recovery is slow (as indicated by the ion temperature measured inside the pedestal close to the pedestal top), causing long ELM cycles. A pellet arriving during this long recovery phase does not succeed in triggering, even when arriving $36 \mathrm{~ms}$ after the previous ELM.
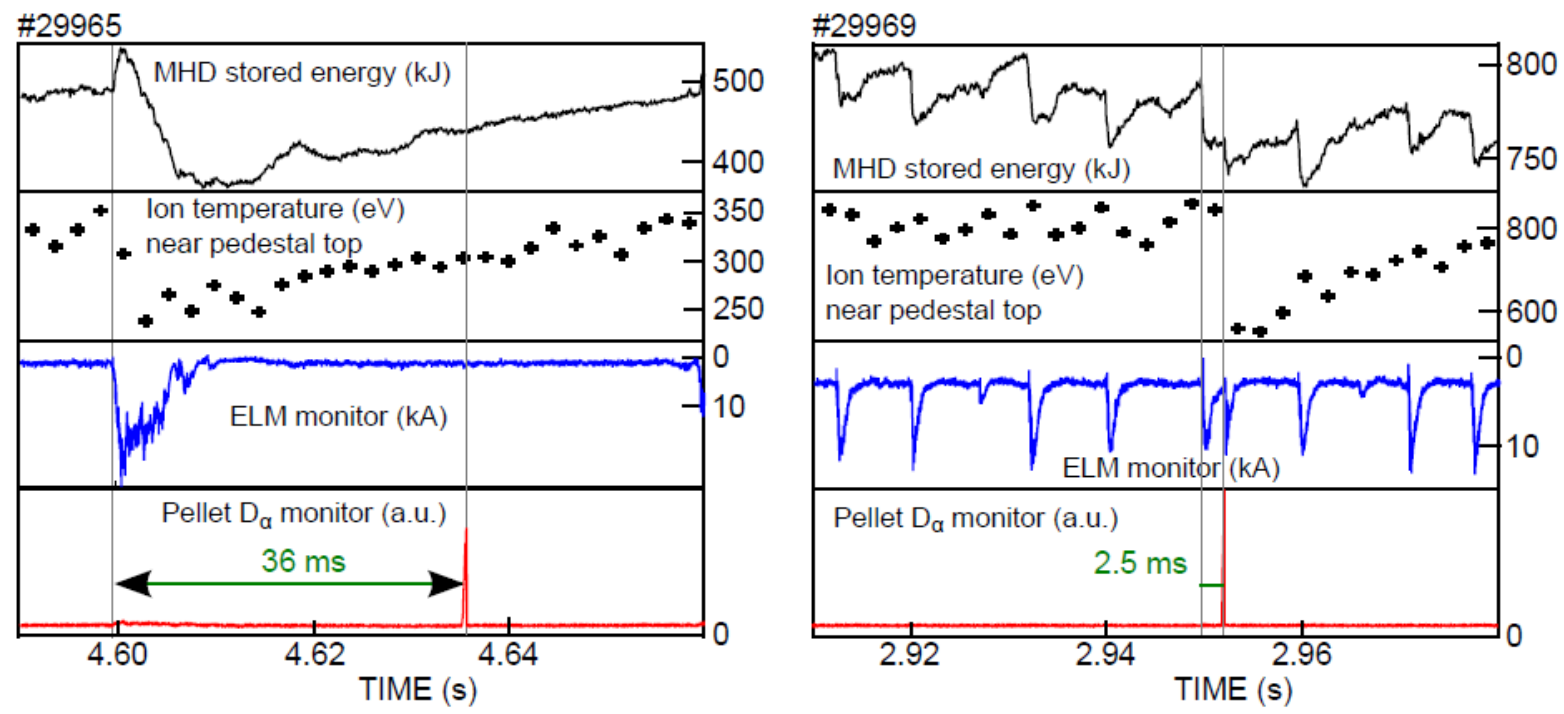

Figure 12: Most extreme cases observed for triggering lag times. In the ITER baseline scenario, suffering from low spontaneous ELM rates and becoming prone to impurity accumulation, attempting to enforce sufficient ELM activity fails; the pellet arriving $36 \mathrm{~ms}$ after the start of the previous ELM does not succeed in triggering (left). In contrast, a pellet arriving after only $2.5 \mathrm{~ms}$ in the ELM cycle clearly triggers in plasmas with higher confined energy. Nitrogen seeding is required in this scenario applying strong auxiliary heating to provide divertor cooling (right).

Contrary behaviour is found in seeding scenarios applying a high auxiliary heating power, where fast recovery of the pedestal produces short spontaneous ELM cycles. In this regime considerable shortening of the lag time also seemingly takes place. The scenario selected was primarily developed for demonstrating the real-time stabilization of Neoclassical Tearing Modes (NTMs) [43]. It was run with up to $\mathrm{P}_{\mathrm{NI}}=15 \mathrm{MW}$ to reach a high normalized pressure; the divertor has to be cooled by using nitrogen $(\mathrm{N})$ seeding [44], which also improves confinement [45]. Here, a shorter trigger lag time was found, ranging down to $2 \mathrm{~ms}$, as can be seen in plot (B) of figure 11. The right part of figure 12 shows a case from those with the 
shortest time span between a leading spontaneous ELM followed by a triggered one. The characteristics found in AUG-C are again encountered in this regime with high heating power applied in the presence of $\mathrm{N}$. In this scenario, however, some pellets arriving up to about $3 \mathrm{~ms}$ after the start of the previous ELM did not trigger. This is still different to the observation made in AUG-C as shown in plot (C) of figure 11. Here, even a pellet arriving whilst the previous ELM was still on-going triggered another crash and any case with no triggering could not be observed.

\section{Trigger threshold and edge stability considerations}

The key question to be finally answered by these investigations is whether pellet pacing will be fully achievable under ITER conditions or if there are limitations, e.g. for the maximum ELM rate or the minimum power loads induced by triggered ELMs. In case there are significant limitations it can be of interest whether the trigger efficiency can be increased, e.g. by adjusting the pellet perturbation magnitude or by changing the poloidal launching position. There are strong indications reported from DIII-D [46] and JET-ILW [18] that the location of the injection path has a significant influence. However, since the AUG system is restricted to the injection geometry shown in figure 1 , it is currently not possible to vary this parameter. On the other hand, by virtue of the wide range of accessible pellet parameters, it was found that, within a certain range, the pellet perturbation magnitude does not play a major role. It appears that it is sufficient if minimum pellet penetration is attained, since the local pelletinduced perturbation is strong enough to trigger an ELM in all cases, provided that the plasma edge has already reached metastable conditions. Stability conditions, in turn, strongly depend on plasma parameters. While it would be premature to draw a final conclusion from the calculated stability boundaries, we shall report on a preliminary stability analysis of the conditions allowing successful ELM triggering in the following. More detailed experiments with a stronger focus on edge diagnostics are planned for the next campaign of AUG.

A stability analysis (for more details of the procedure see [47]) was done by taking measurements from Thomson scattering (TS), electron cyclotron emission (ECE), charge exchange recombination spectroscopy (CXRS), and lithium-beam data, providing accurate $\mathrm{T}_{e}$, $\mathrm{T}_{i}$ and $\mathrm{n}_{e}$ profiles in the pedestal region and allowing derivation of a full pressure (p) profile. These measurements were mapped onto the radial mid-plane along flux surfaces and then fitted with the commonly used modified hyperbolic tangent function. The kinetic profiles, as well as the data from magnetic field and flux difference measurements, were then used to provide inputs to the CLISTE Grad-Shafranov solver [48] in order to calculate a selfconsistent plasma equilibrium including full current density profiles. This equilibrium was then refined by using the HELENA fixed boundary equilibrium solver and used as a basis for stability calculations with the ILSA linear MHD code. The code was run in ideal MISHKA-1 mode to calculate the growth rates of the toroidal mode numbers $n=1-20$. As this analysis assumes axisymmetric equilibria, it cannot describe the locally perturbed plasma after an incoming pellet. While the calculations presented characterize the stability of the plasma prior to a spontaneous ELM or the arrival of a pellet, the impact of the pellet itself could be determined in the future by using non-axisymmetric stability codes such as JOREK $[12,13]$. However, it is interesting to explore whether triggering ELMs is only possible if the plasma is close to the stability boundary for axisymmetric equilibria.

The first case analysed is the discharge shown in figures 6 and 7, run in AUG-W without $\mathrm{N}$ seeding at $\mathrm{I}_{\mathrm{P}}=1.0 \mathrm{MA}, \mathrm{B}_{\mathrm{t}}=2.5 \mathrm{~T}$ and $\mathrm{P}_{\mathrm{NI}}=5 \mathrm{MW}$, the gas puff rate was being set at $6.7 \times 10^{21} \mathrm{D} / \mathrm{s}$. The resulting spontaneous ELM frequency was scattered between 30 and 
$130 \mathrm{~Hz}$. ELMs could be triggered after a lag time of $7 \mathrm{~ms}$, which corresponds to the interELM time of the spontaneous ELMs occurring with the highest frequency in this discharge, i.e. $140 \mathrm{~Hz}$. The edge temperature profiles could not be ideally diagnosed since no radial sweep of the plasma could be made to gather TS data and improve the quality of edge measurements. However, it is the best-diagnosed discharge of this type available. The electron temperature profiles presented in figure 13 (a) are, therefore, preliminary until dedicated experiments become available in the next campaign of AUG. Three time slices are presented in this analysis. All data were fitted by means of coherent ELM-averaging over $3 \mathrm{~ms}$ to achieve a minimum data quality and because edge $T_{i}$ measurements are integrated over $2.2 \mathrm{~ms}$. The first interval is taken just before the ELM crash ("-1.5 ms", black). For the second one ("+5.5 ms", blue), data from $4 \mathrm{~ms}$ to $7 \mathrm{~ms}$ after the ELM crash were used, this corresponding to a plasma in which ELM triggering is not yet possible. The last set of profiles shown in figure 13 ("+8.5 ms", red) was taken between $7 \mathrm{~ms}$ and $10 \mathrm{~ms}$ after the ELM crash, where triggering just became possible. While all red profiles almost reach the high pre-ELM values of the black profiles, $5.5 \mathrm{~ms}$ after the ELM the temperature, density and, therefore, pressure pedestals are still very shallow. The pressure gradient (d) is at less than $50 \%$ of its maximum value and the edge current density (e) is also very low. Therefore, it is not surprising that it is only possible to trigger ELMs in the later time interval. While it would be interesting to correlate the profile evolution with the possibility of successful ELM triggering, the quality of the data is not yet sufficient to perform a detailed ELM cycle analysis like that presented in reference [40].
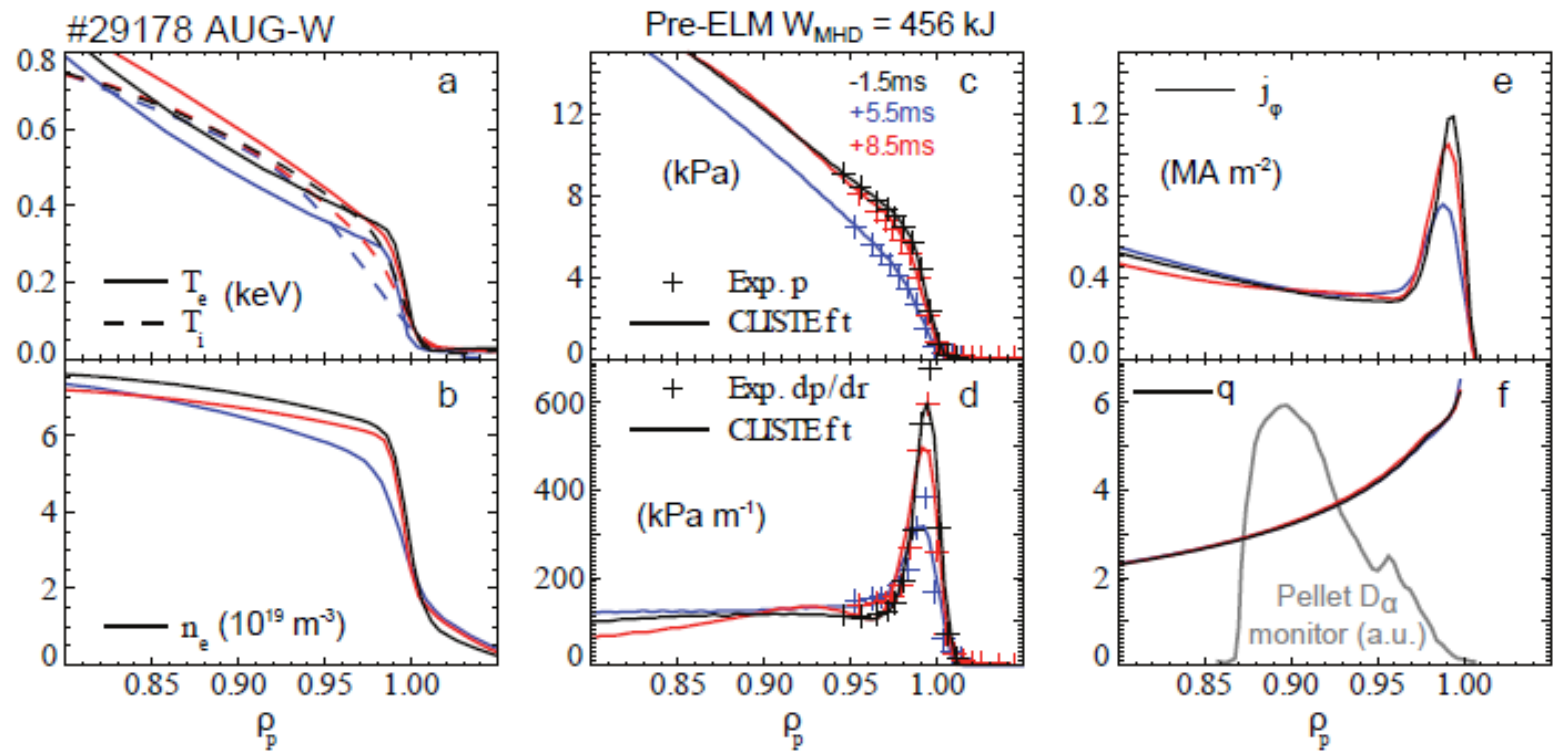

Figure 13: Profiles at different time points in the ELM cycle in AUG-W: electron and ion temperatures (a), electron density (b), total pressure (c), total pressure gradient (d), toroidal current density at LFS (e), safety factor $q$ and mapped pellet ablation ( $f$ ) 

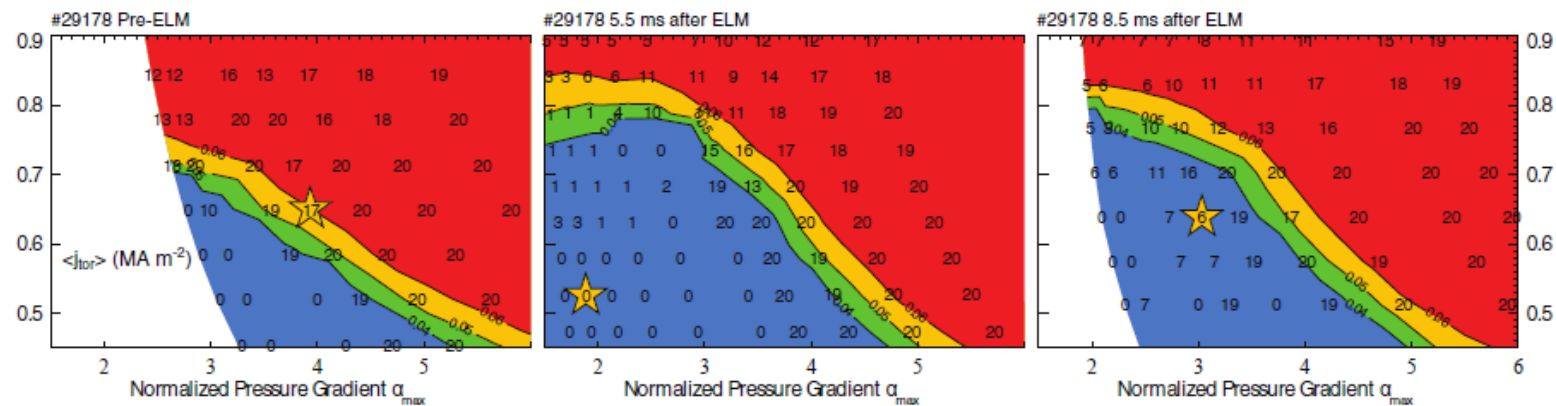

Figure 14 : Stability diagrams of an unseeded AUG-W plasma before a spontaneous type-I ELM (left), $5.5 \mathrm{~ms}$ after the ELM crash (middle) and $8.5 \mathrm{~ms}$ after the ELM crash (right)

However, a preliminary stability analysis was made at the three selected time points in the ELM cycle, as shown in figure 14. Stability is shown as a function of two destabilizing quantities, the pressure gradient and the current density. $\alpha_{\max }$ is the maximum normalized edge pressure gradient (defined in [49]). The maximum toroidal edge current density is fluxsurface averaged, also serving as a proxy for the magnetic shear. The positions of the numbers mark the grid points of the $j-\alpha$ grid, while the numbers themselves correspond to the dominant toroidal mode. The critical value for stability chosen here is $\gamma / v_{\mathrm{A}}<0.06$, where $\gamma$ is the growth rate of the mode and $v_{\mathrm{A}}$ the Alfven frequency. While this value is somewhat arbitrary, it accurately separates the region in which the ILSA code finds clear unstable modes from one where it does not find any instabilities at all or only numerically unstable solutions. The diagrams confirm what could already be expected from the profiles in figure 13: the pre-ELM time point is located on the stability boundary, while the other two equilibria are considered to be stable. However, the equilibrium from $7-10 \mathrm{~ms}$ in the recovery phase is only $20 \%$ below the stability boundary. Between $4 \mathrm{~ms}$ and $7 \mathrm{~ms}$ after the ELM crash a pressure gradient increase of more than $100 \%$ and a large current density increase would be necessary to reach the boundary. In the reference case analysed for the full-metal machine, ELM triggering by means of pellets seemingly becomes possible when the edge pressure gradient and current density recover to approximately $20 \%$ below their pre-ELM values and the ideal MHD stability boundary for axisymmetric equilibria.

Seeding nitrogen in the divertor significantly decreases the lag time necessary for successful ELM triggering to $1.5 \mathrm{~ms}-3 \mathrm{~ms}$. Figure 15 shows the edge profiles of the nitrogen-seeded discharge briefly discussed in section 6 , which was run at $1 \mathrm{MA},-2.6 \mathrm{~T}$ and heated with $10 \mathrm{MW}$ NBI, 1.5 MW ECRH and 1.5 MW ICRH. While the ELMs mostly had a frequency between 80 and $130 \mathrm{~Hz}$, a few sporadic spontaneous ELMs did occur only 2 ms after the previous one. The black profiles were fitted by using data from a $2.9 \mathrm{~ms}$ time interval just before a spontaneous ELM, and the red ones by using data from $3 \mathrm{~ms}$ after the ELM crash, when ELM triggering is possible. 3 ms after the ELM crash $T_{i}$ and $n_{e}$ have almost fully recovered to their pre-ELM values, while the pedestal top electron temperature is still $20 \%$ lower than before a spontaneous ELM. This leads to a slightly lower pedestal top pressure and $20 \%$ lower edge pressure gradient and current density. These results are in line with those obtained in non-seeded discharges, where ELM triggering becomes possible when the pedestal has recovered to values similar to those of the pre-ELM pedestal.

In addition, a discharge from the AUG-C phase was analysed for comparison. It was run at $I_{P}$ $=1.0 \mathrm{MA}, \mathrm{B}_{\mathrm{t}}=2.6 \mathrm{~T}, \mathrm{P}_{\mathrm{NI}}=5 \mathrm{MW}$ and $1.3 \mathrm{MW}$ ion cyclotron heating. Fuelled at only $0.3 \times 10^{21} \mathrm{D} / \mathrm{s}$, the plasma had an ELM frequency between 45 and $55 \mathrm{~Hz}$ with only very few ELMs slightly exceeding $60 \mathrm{~Hz}$. Unlike the nitrogen-seeded discharge in tungsten, no sporadic spontaneous ELMs occurred immediately after a previous event. Reliable ELM- 
triggering could already be achieved 1.6 ms after the previous ELM crash, much more quickly than the fastest occurring natural ELM. No indication of the presence of a lag time was found in this case.
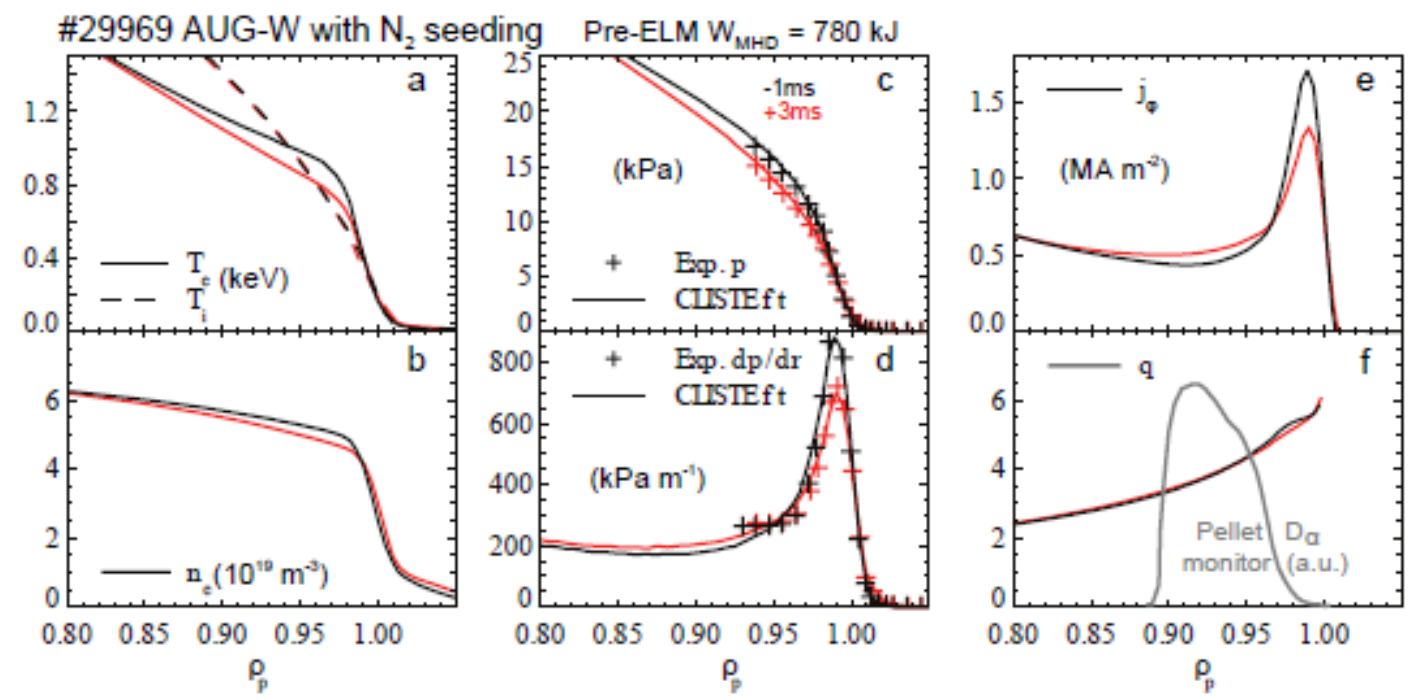

Figure 15: Profiles at different time points in the ELM cycle in AUG-W with nitrogen seeding in the divertor: electron and ion temperatures (a), electron density (b), total pressure (c), its gradient (d), toroidal current density at the LFS (e), safety factor $q$ and mapped pellet ablation (f)
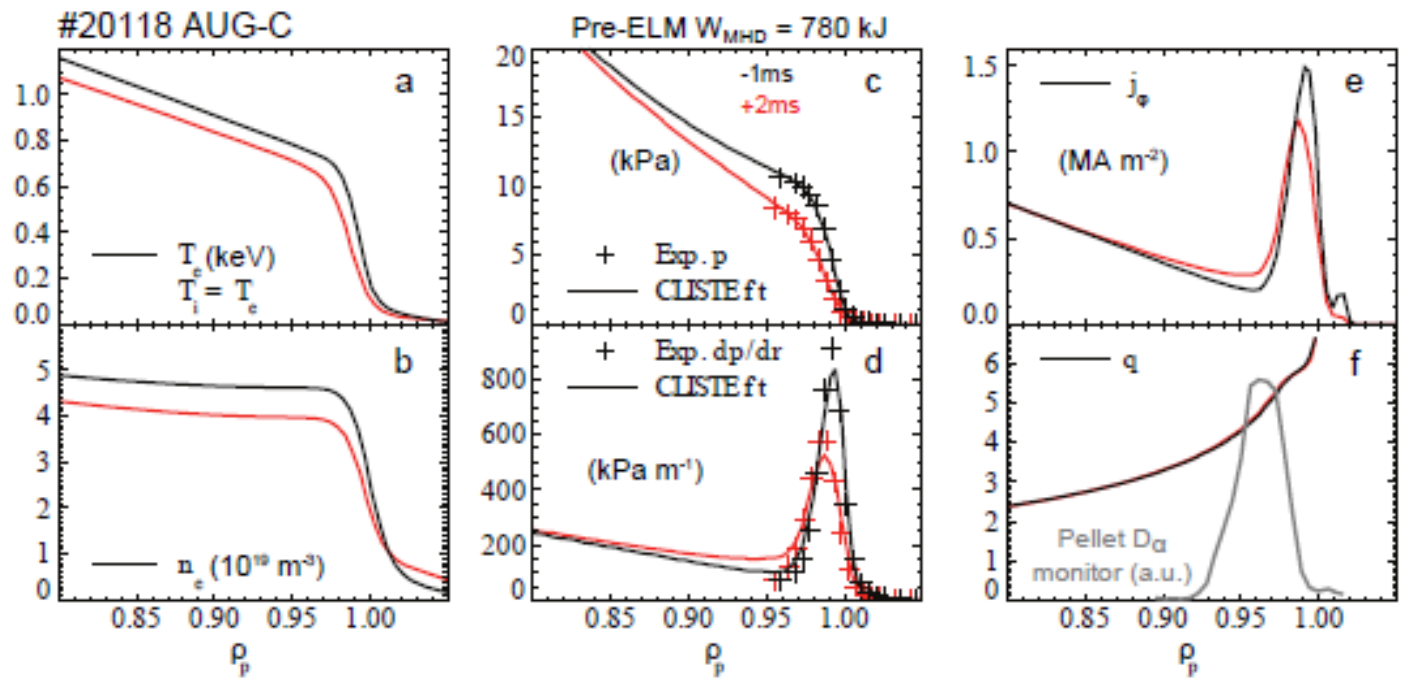

Figure 16: Profiles at different time points in the ELM cycle in AUG-C: electron and ion temperatures (a), electron density (b), total pressure (c), its gradient (d), toroidal current density at the LFS (e), safety factor $q$ and mapped pellet ablation ( $f$ )

The corresponding kinetic profiles are presented in figure 16. The black profiles ("-1 ms") were taken prior to an ELM and the red ones (“+2 ms") were ELM-synchronized between 1.5 and $3 \mathrm{~ms}$ after the crash. Since the edge CXRS system was not yet installed when the PFCs were still partly made of uncoated graphite, it was assumed that $T_{i}=T_{e}$. While the ELMinduced drops in the temperature are much smaller than in the $\mathrm{W}$-coated reference case, the 
pedestal density 2 ms after a spontaneous ELM crash is still much lower than the pre-ELM value. The pressure profile is not yet recovered and the local edge current density still needs to increase by roughly $25 \%$ before it reaches its pre-ELM value.

Results from the stability analysis seem to indicate that ELM triggering is only possible if the plasma is close to the pre-ELM values of the equilibrium quantities. A pressure gradient of roughly $25 \%$ below the pre-ELM value already seems to be sufficient for successful ELM triggering, but the precise value still remains to be determined. This is consistent with results from DIII-D, where the pressure gradient drops by $20 \%$ during the ELM crash, at which point ELM triggering is already successful [40]. Therefore, the magnitude of the ELM and the subsequent recovery phase are the decisive factors determining whether triggering can be achieved or not. The smaller ELM crash and the faster recovery of the pressure profile when seeding nitrogen in a full-metal machine [50] could explain the shorter lag time.

Further experiments aiming at comparison of the threshold for ELM triggering in unseeded and N-seeded discharges in AUG-W will be conducted in the next experimental campaign of AUG. Dedicated discharges optimized for edge measurements are expected to yield detailed results on the exact shape of the critical kinetic profiles necessary for ELM triggering. While discharges in AUG-C cannot be repeated, it might be possible to simulate carbon walls by seeding methane.

\section{Efficient particle fuelling and high density operation with benign ELM behaviour}

Operating AUG with a fully W-covered wall significantly reduced the pellet ELM trigger potential with respect to AUG-C. This is probably a disadvantage not to be overcome for any pacing and mitigation application. On the other hand, this changed behaviour also bears fruit when employing pellets for particle refuelling tasks and pursuing operation at high density. The advantage of pellet fuelling in the absence of strong ELM activity has already been demonstrated when applying magnetic perturbation generated by active in-vessel saddle coils for ELM mitigation [51]. High-density operation without confinement degradation and benign ELMs can also be achieved without externally imposed magnetic perturbations. Abstaining from any saddle coil activation, we established an operational scenario for core densities far beyond $\mathrm{n}_{\mathrm{Gw}}$. Figure 17 shows an example where the pellet system's full actual capability was employed for a fuelling attempt. The launcher delivers the largest available pellets at a rate of $70 \mathrm{~Hz}$; this is equivalent to an averaged flux at the centrifuge exit of about $2.6 \times 10^{22} \mathrm{D} / \mathrm{s}$. A pellet speed of $566 \mathrm{~m} / \mathrm{s}$ was chosen as a trade-off between maximum pellet penetration and pellet mass erosion during transfer. The entire ice reservoir from the storage cryostat was consumed, providing a train that lasted $1.35 \mathrm{~s}$. The target plasma had $\mathrm{I}_{\mathrm{P}}=1.0 \mathrm{MA}$, $\mathrm{B}_{\mathrm{t}}=2.55 \mathrm{~T}, \mathrm{q}_{95}=4.65, \kappa=1.70, \delta^{\mathrm{u}}=0.11, \delta^{\mathrm{l}}=0.39$ and $\mathrm{P}_{\mathrm{NI}}=7.5 \mathrm{MW}$. Avoiding impurity accumulation necessitated additional core heating. With both ECRH and ICRH initially provided, shortly after the pellets set in ECRH was switched off for machine protection to avoid strong power reflection at high-density cut-off layers. Hence, during the entire highdensity phase and also after the pellet phase, core heating is solely contributed by ICRH. Furthermore, a minimum residual gas puff rate was required.

Driven by the pellet train, a high-density phase is reached and kept until all the pellets are consumed. The high-density regime can be reliably and reproducibly established; the pelletgenerated density increase is fully reversible. After termination of the pellet sequence the plasma returns to the initial conditions and the discharge can be safely ramped down. During the high-density phase, the normalized plasma pressure slightly drops to about 1.6 from a value of about 1.7 , found in the initial and later reference phases. Line-averaged densities 
significantly beyond $\mathrm{n}_{\mathrm{Gw}}$ are achieved during the high-density phase. Again, as already reported in the previous study with in-vessel coil activation [51], this is due to the peaked density profiles generated. The Greenwald density imposes a limit, but only on the edge density. With the profiles becoming more peaked inside the pedestal, a core region extending further and further outwards attains densities beyond $\mathrm{n}_{\mathrm{Gw}}$. Hence, the "Greenwald limit" can be regarded as an edge limit which can easily be overcome by pellet injection. Typical profiles are found with a strong density gradient extending from the edge into the core towards about the pellet burn-out point as measured by the pellet tracking system; beyond the pellet penetration depth the density remains almost constant. There is also some indication of enhanced particle confinement when reaching the highest densities. Then, sustainment times of pellet-deposited particles, as determined from the density decline after every single pellet, increase by a factor of up to about 3 with respect to the initial values.

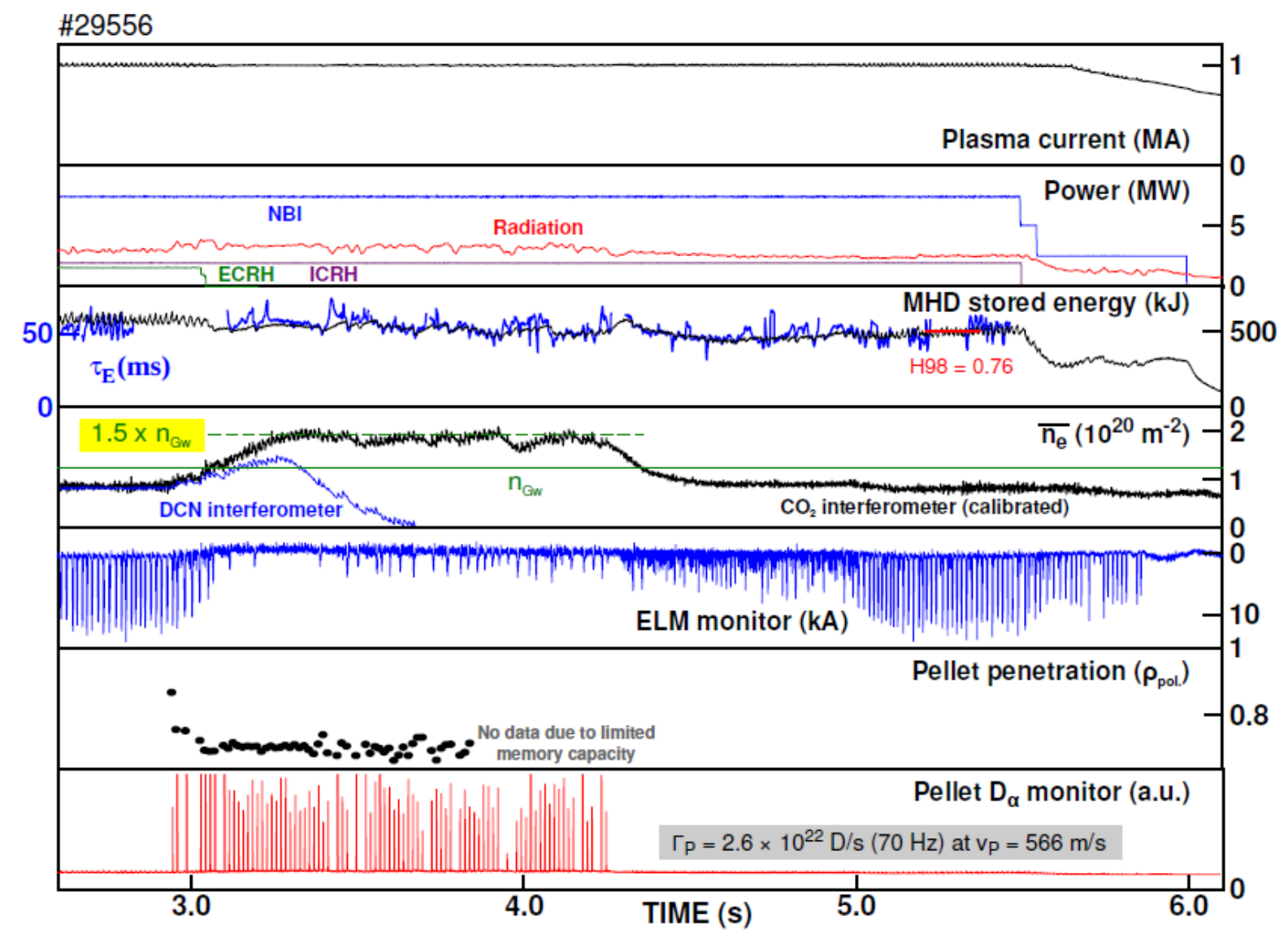

Figure 17: High-density operation without confinement degradation achieved by pellet fuelling. Densities far beyond the Greenwald values were achieved with benign ELM behaviour.

As can already be seen from figure 17, the ELM behaviour becomes benign during the high density phase. Regular strong type-I ELMs present before and after this phase disappear, while the remaining ELMs are essentially type-III ELMs with a few type-I ELMs of low magnitude in between. It is worth mentioning that, for the plasma scenario chosen for this approach, activation of the in-vessel coils did not show any significant impact.

The approach reported here was just a first test demonstrating that pellet fuelling has become even more suited in AUG-W. Further optimization of the overall performance seems 
probable. The performance with respect to energy confinement, although maintained during the high-density phase, is only modest. The moderate heating power and residual gas puffing applied make confinement in the reference phase (indicated in figure 17) only about 0.76 times the value predicted by the ITERH98P $(y, 2)(H 98)$ scaling [52]. In the discharge shown, a constant gas flux of $3 \times 10^{22} \mathrm{D} / \mathrm{s}$ was applied. Gradual reduction of the puffing rate resulted first in a slight enhancement of the density and energy. Further reduction causes a rollover of both parameters as first 3,2 and then 2,1 NTM activity sets in, causing some confinement deterioration. In the absence of any external gas puffing, a short initial high-performance phase with $\mathrm{H} 98=1.12$ is observed terminated by impurity accumulation, finally causing a radiative collapse. This indicated that there is still room for improvement. Operational experience from AUG-W and JET-ILW [20] shows that there is always the need for substantial gas puff. In cases where only a modest heating power was applied, as in the fuelling attempt presented here, energy confinement significantly lower than in bestperforming $\mathrm{C}$ wall discharges was found. However, the confinement and H98 values in the vicinity of 1 were recovered when sufficiently high heating power was applied. Hence, it seems plausible that operation at high densities and even better confinement can be achieved by applying pellet fuelling in high-power scenarios, eventually requiring operation at higher pellet speed to keep sufficient penetration. Preparations are underway to make appropriate efforts in the upcoming campaign at AUG.

A careful analysis showed the energy confinement time $\tau_{\mathrm{E}}$ does not change during and after the high density phase (indicated by a solid blue line in figure 17). No indication is found for a confinement increase as predicted by the H98 scaling. This scaling becomes inappropriate for evaluating the plasma performance once densities beyond about $0.85 \times \mathrm{n}_{\mathrm{Gw}}$ are achieved.

\section{Summary and outlook}

With the change-over from a $\mathrm{C}$ to an all-metal wall, ELM triggering and pacing have become more intricate. This observation, previously made at JET for pellet pacing [18], has now been confirmed at AUG for two different methods, viz. magnetic and pellet triggering. This change of first-wall material correlates with increased collisionality in the pedestal region due to operational conditions resulting in higher densities and lower temperatures in the edge. This claim is supported by the similar collisionalities and ELM trigger responses in AUG-C and Nseeded AUG-W.

ELM triggering by pellets in all-metal-wall tokamaks entails a lag time. It was first spotted at JET with an indication that the poloidal launch position and pellet parameters can have an influence on it. While the first observation still needs to be further investigated, the latter turned out to be relevant only in respect of a pellet arriving under sufficiently viable conditions to penetrate fairly deeply into the edge pedestal. Careful investigations at AUG show that pellet parameter do not play a role since variations of speed and mass did not show any effect. The pellet particle ablation rate per second, $\mathrm{dN} / \mathrm{dt}$, can be taken as a measure for estimating how much the magnitude of the resulting pellet-imposed local perturbation can be varied by the pellet parameters at hand. For scaling the ablation $\mathrm{dN} / \mathrm{dt} \sim \mathrm{m}_{\mathrm{P}}{ }^{0.35} \times \mathrm{v}_{\mathrm{P}}{ }^{0.18}$ can be adopted as in a numerical hybrid code [53]. This was found to correctly reproduce all the basic features of inboard launched pellets in AUG [54]. This results in a maximum ratio between the strongest (large, fast) and weakest (small, slow) perturbation of about 1.8, this being inadequate to shorten the trigger lag time. Changing plasma parameters can alter the lag time; stronger heating and possibly the presence of $\mathrm{N}$ can considerably shorten it. On the other hand, regimes characterized by low heating power and low ELM rates provide little 
potential for pellet ELM triggering. At the beginning of the pedestal build-up phase in AUG$\mathrm{W}$, when the pressure gradient has only recovered to 50\% of its pre-ELM value, triggering is not possible. In all of the cases analysed (AUG-W and AUG-C) in which successful triggering was achieved, the pressure gradient and edge current density were closer to their pre-ELM values. This is not in disagreement with the DIII-D results, which showed that an increase of the ELM frequency by a factor 12 in relation to the spontaneous ELM frequency could be achieved [40], since the pressure gradient and current density prior to a triggered ELM were greater than $75 \%$ of their pre- spontaneous ELM values. These results suggest that ELM triggering can only be achieved when the plasma edge has recovered obviously enough to the values observed prior to the occurrence of a spontaneous ELM, but the exact threshold remains to be determined. This calls for further investigations that focus on the correlation between plasma conditions and trigger response. Furthermore, experiments have to provide clear and well-documented cases, both with and without trigger response in a variety of scenarios, to provide a complete experimental basis for detailed modelling efforts. The goal of such an experiment and comparison with theory must be to determine the underlying mechanism causing the reduced capability to trigger ELMs, finally allowing a sound prediction for ITER.

Last but not least, the topic of ELM mitigation and especially the possible impact of pacing on the peak fluency during an ELM have to be further examined. So far, investigations in allmetal-wall tokamaks were restricted to cases with very modest enhancement of the ELM frequency. Comparison with the latest encouraging mitigation demonstration from DIII-D [40] calls for a similar experiment to be performed under all-metal-wall conditions. For various reasons, this can best be done by using HFPI at JET once it has been relocated for better inboard pellet delivery reliability.

The reduced capability of pellets to trigger an ELM in an all-metal-wall environment has the potential to cause serious concern as regards pellets being used as a tool for ELM pacing and mitigation. However, this changed behaviour can yield in turn a remarkable improvement for pellets applied as a fuelling tool. A first demonstration already succeeded in showing a good performance in combination with benign ELM behaviour, even without externally applied magnetic perturbations. Naturally, there is still room for further improvements in performance, but first attempts raise the hope that this could induce a renaissance of pellet fuelling when using inboard launch in an all-metal-wall tokamak.

\section{References}

[1] S.L. Milora, W.A. Houlberg, L.L. Lengyel and V. Mertens, 1995, Nucl. Fusion 35, 657

[2] T.H. Osborne et al, 2001, Phys. Plasmas 8, 2017

[3] T.H. Osborne et al, 2001, J. Nucl. Mater. 290-293, 1013.

[4] H. Zohm et al., 2013, Nucl. Fusion 53, 073019.

[5] D.J. Campbell et al., 2012, Proc. 24th Int. Fusion Energy Conf. (San Diego, CA, 2012)

(Vienna: IAEA), ITR/P1-18

and http://www-naweb.iaea.org/napc/physics/FEC/FEC2012/index.htm

[6] J.W. Connor, 1998, Plasma Phys. and Control. Fusion 40, 191.

[7] P.B. Snyder and H.R. Wilson, 2002, Contributions to Plasma Phys. 4, 258.

[8] A. Kirk et al., 2004, Plasma Phys. Control. Fusion 46, A187.

[9] R.J. Groebner et al., 1998, Plasma Phys. Control. Fusion 40, 673.

[10] H.R. Wilson and S.C. Cowley, 2004, Phys. Rev. Lett. 92, 175006.

[11] T.H. Osborne et al, 2000, Plasma Phys. Control. Fusion 42, 1. 
[12] S. Futatani et al., 2014, Nucl. Fusion 54, 073008.

[13] G.T.A. Huysmans et al., 2009 Plasma Phys. Control. Fusion 51 ,124012

http://iopscience.iop.org/0029-5515/53/12/123023/refs/9/article

[14] E. de la Luna et al., 2012, Proc. 24th Int. Fusion Energy Conf. (San Diego, CA, 2012)

(Vienna: IAEA) EX/6-1

and www-naweb.iaea.org/napc/physics/FEC/FEC2012/index.htm

[15] A. Loarte et al., 2014, Nucl. Fusion 54, 033007.

[16] P.T. Lang et al, 2004, Nucl. Fusion 44665

[17] L.R. Baylor et al., 2010, Proc. 37th EPS Conf. on Controlled Fusion and Plasma Physics

(Dublin, Ireland, 2010) P2.117

and http://ocs.ciemat.es/EPS2010PAP/pdf/P2.117.pdf

[18] P. T. Lang et al., 2013, Nucl. Fusion 53, 073010

[19] L.R. Baylor et al., 2013, Phys. Rev. Lett. 110, 245001

[20] R. Neu et al. 2013, Phys. Plasmas 20,056111

[21] M.N.A. Beurskens et al, 2012, Proc. 24th Int. Fusion Energy Conf. (San Diego, CA, 2012) (Vienna: IAEA) EX/P7-20

and www-naweb.iaea.org/napc/physics/FEC/FEC2012/index.htm

[22] A. W. Degeling et al, 2003, Plasma Phys. Control. Fusion 45, 1637

[23] A. Geraud et al, 2012, 27th SOFT Conf. (Liège, Belgium, September 2012) P1.31 and http://sciconf.org/soft2012/ip/topic/c/session/p1/paper/31

[24] G. Kocsis et al., 2007, Nucl. Fusion 47, 1166

[25] P. T. Lang et al., 2013, Nucl. Fusion 53, 043004

[26] J. Paméla et al., 2003, Nucl. Fusion 43, 1540

[27] A. Geraud et al. 2007, Fusion Eng. Des. 82, 2183

[28] B. Plöckl et al., 2011, Fusion Engineering and Design 86,1022

[29] U. Stroth et al., 2013, Nucl. Fusion 53, 104003

[30] W. Suttrop et al., Proc. 40th EPS Conf. on Controlled Fusion and Plasma Physics

(Espoo, Finland, 2013) P4.117

[31] G. Raupp et al., 2009, Fusion Engineering and Design 84,1575

[32] A. Kallenbach, et al., Plasma Phys. Control. Fusion 52 (2010) 055002 doi:10.1088/07413335/52/5/055002

[33] W. Schneider et al., Fusion Engineering and Design, 48 (2000), 127

[34] B. Plöckl and P.T. Lang, 2013, Rev. Sci. Instrum., 84, 103509

[35] P.T. Lang et al., 2004, Plasma Phys. Control. Fusion 46, L31

[36] T. Szepesi et al., 2009, Plasma Phys. Control. Fusion 51, 125002

[37] F.M. Poli et al., 2010, Nucl. Fusion 50, 025004

[38] P.T. Lang et al., Plasma Phys. Control. Fusion 48 (2006) A141, doi:10.1088/07413335/48/5A/S13

[39] A. Burckhart et al., Plasma Physics Contr. Fusion 52, 105010 (2010) and

A. O. Burckhart, "Different ELM regimes at ASDEX Upgrade and their linear stability analysis”, PhD. Thesis TU Munich; IPP Report 17/41 Dissertation; http://edoc.mpg.de/display.epl?mode=doc\&id=672912

[40] L.R. Baylor et al., Phys. Rev. Lett. 110, 2013, 245001

[41] J. Schweinzer et al., Proc. 40th EPS Conf. on Controlled Fusion and Plasma Physics

(Espoo, Finland, 2013) P2.134

[42] A. Kallenbach, et al., J. Nucl. Mater. 337-339, 2005, 732

[43] M. Reich et al., Proc. 40th EPS Conf. on Controlled Fusion and Plasma Physics (Espoo,

Finland, 2013) P2.151

[44] A. Kallenbach, et al., 2012, Nucl. Fusion 52, 122003 
[45] J. Schweinzer et al., 2011, Nucl. Fusion 51, 113003

[46] L.R. Baylor et al., 2007, Nucl. Fusion 47, 1598

[47] C. Konz et al., Proc. 36th EPS Conf. on Controlled Fusion and Plasma Physics (Sofia, Bulgaria, 2009) P1.152

[48] P. McCarthy, ASDEX Upgrade Team, 2012, Plasma Phys. Control. Fusion 54, 015010, doi:10.1088/0741-3335/54/1/015010

[49] R.L. Miller, 1998, Phys. Plasmas 5, 973

[50] P.A. Schneider et al., Proc. 40th EPS Conf. on Controlled Fusion and Plasma Physics (Espoo, Finland, 2013) P4.106

[51] P. T. Lang et al., 2012, Nucl. Fusion 52, 023017

[52] ITER Physics Basis Expert Groups on Confinement and Transport and Confinement Modelling and Database, ITER Physics Basis Editors 1999 Nucl. Fusion 39, 2175

and http://iopscience.iop.org/0029-5515/52/2/023017/refs/23/article

[53] K. Gal et al., 2008, Nucl. Fusion 48, 085005

and http://iopscience.iop.org/0029-5515/48/8/085005/

[54] E. Belonohy et al., 2008, Nucl. Fusion 48, 065009

and http://iopscience.iop.org/0029-5515/48/6/065009/ 